\title{
THE THERMAL EVOLUTION OF ICES IN THE ENVIRONMENTS OF NEWLY FORMED STARS: THE $\mathrm{CO}_{2}$ DIAGNOSTIC
}

\author{
A. M. Cook $^{1,5}$, D. C. B. Whittet ${ }^{1}$, S. S. Shenoy ${ }^{2,5}$, P. A. Gerakines ${ }^{3}$, D. W. White ${ }^{3}$, and J. E. Chiar ${ }^{4}$ \\ ${ }^{1}$ New York Center for Astrobiology and Department of Physics, Applied Physics \& Astronomy, Rensselaer Polytechnic Institute, \\ 110 Eighth Street, Troy, NY 12180, USA \\ ${ }^{2}$ Spitzer Science Center, Mail Code 220-6, California Institute of Technology, Pasadena, CA 91125, USA \\ ${ }^{3}$ Department of Physics, University of Alabama at Birmingham, 310 Campbell Hall, 1300 University Boulevard, Birmingham, AL 35294, USA \\ ${ }^{4}$ SETI Institute, 189 Bernardo Avenue, Mountain View, CA 94043, USA \\ Received 2010 July 22; accepted 2011 February 4; published 2011 March 11
}

\begin{abstract}
Archival data from the Infrared Spectrometer of the Spitzer Space Telescope are used to study the $15 \mu \mathrm{m}$ absorption feature of solid $\mathrm{CO}_{2}$ toward 28 young stellar objects (YSOs) of approximately solar mass. Fits to the absorption profile using laboratory spectra enable categorization according to the degree of thermal processing of the ice matrix that contains the $\mathrm{CO}_{2}$. The majority of YSOs in our sample (20 out of 28) are found to be consistent with a combination of polar $\left(\mathrm{H}_{2} \mathrm{O}\right.$-rich) and nonpolar (CO-rich) ices at low temperature; the remainder exhibit profile structure consistent with partial crystallization as the result of significant heating. Ice-phase column densities of $\mathrm{CO}_{2}$ are determined and compared with those of other species. Lines of sight with crystallization signatures in their spectra are found to be systematically deficient in solid-phase $\mathrm{CO}$, as expected if $\mathrm{CO}$ is being sublimated in regions where the ices are heated to crystallization temperatures. Significant variation is found in the $\mathrm{CO}_{2}$ abundance with respect to both $\mathrm{H}_{2} \mathrm{O}$ (the dominant ice constituent) and total dust column (quantified by the extinction, $A_{V}$ ). YSOs in our sample display typically higher $\mathrm{CO}_{2}$ concentrations (independent of evidence for thermal processing) in comparison to quiescent regions of the prototypical cold molecular cloud. This suggests that enhanced $\mathrm{CO}_{2}$ production is driven by photochemical reactions in proximity to some YSOs, and that photoprocessing and thermal processing may occur independently.
\end{abstract}

Key words: astrochemistry - dust, extinction - evolution - infrared: ISM - ISM: molecules - stars: pre-main sequence

Online-only material: color figures

\section{INTRODUCTION}

Icy material is the dominant carrier of the $\mathrm{CNO}$ group of chemical elements from interstellar clouds to protoplanetary disks. Understanding the nature and evolution of this material is thus an important astrophysical and astrobiological goal, with significance for models of interstellar chemistry, planet formation, and the origin of comets and other icy bodies in the solar system. The absorption feature identified with the bending mode of solid carbon dioxide $\left(\mathrm{CO}_{2}\right)$ near $15 \mu \mathrm{m}$ has proven to be particularly useful in this regard, providing insight into the thermal evolution as well as the composition of the ices. Absorption at this wavelength is widely observed in spectra obtained with the Infrared Space Observatory (ISO) and the Spitzer Space Telescope for lines of sight that contain substantial columns of cold interstellar dust and molecular gas in our Galaxy. Detections have been made toward both dust-embedded young stellar objects (YSOs; Gerakines et al. 1999; Gibb et al. 2000, 2004; Nummelin et al. 2001; Boogert et al. 2004; Watson et al. 2004; Pontoppidan et al. 2005, 2008; Zasowski et al. 2009) and background field stars located behind dense molecular clouds (Bergin et al. 2005; Knez et al. 2005; Whittet et al. 2007, 2009). These studies demonstrate that $\mathrm{CO}_{2}$ is a ubiquitous component of the ices that reside upon dust grains inside the clouds. It is, indeed, expected that $\mathrm{CO}_{2}$ will form efficiently in the environments of YSOs, where a source of activation energy needed to drive reactions

\footnotetext{
5 Current address: NASA Ames Research Center, Mail Stop 245-6, Moffett Field, CA 94035, USA.
}

such as $\mathrm{CO}+\mathrm{O} \rightarrow \mathrm{CO}_{2}$ and $\mathrm{CO}+\mathrm{OH} \rightarrow \mathrm{CO}_{2}+\mathrm{H}$ is readily available. Laboratory experiments demonstrate, for example, that $\mathrm{CO}_{2}$ is produced when ice mixtures containing $\mathrm{H}_{2} \mathrm{O}$ and $\mathrm{CO}$ are subjected to $\mathrm{UV}$ radiation in conditions analogous to those in a molecular cloud near a hot embedded star (e.g., d'Hendecourt et al. 1986). However, the presence of $\mathrm{CO}_{2}$ in lines of sight toward background field stars shows that $\mathrm{CO}_{2}$ is also formed in quiescent regions of molecular clouds that lack a local energy source, presumably by grain surface reactions that proceed at very low temperature (e.g., Whittet et al. 1998; Langer et al. 2000; Pontoppidan et al. 2008). Recent laboratory work demonstrates the formation of $\mathrm{CO}_{2}$ from $\mathrm{CO}$ and $\mathrm{OH}$ at temperatures as low as $10 \mathrm{~K}$ (Oba et al. 2010).

Since YSOs are born in regions that were once quiescent, observations of field stars provide insight into initial conditions prior to thermal and/or photochemical evolution driven by star formation. The observations show that ices in quiescent clouds are composed primarily of three species, $\mathrm{H}_{2} \mathrm{O}, \mathrm{CO}$, and $\mathrm{CO}_{2}$, of which only $\mathrm{CO}$ is present in concentrations consistent with adsorption of molecules formed in the gas phase. At intermediate densities, the most abundant reactive species attaching to grain surfaces are $\mathrm{H}, \mathrm{O}$, and $\mathrm{CO}$, from which $\mathrm{H}_{2} \mathrm{O}$ and $\mathrm{CO}_{2}$ appear to form in tandem by hydrogenation of $\mathrm{O}$ and oxidation of $\mathrm{CO}$ to produce a "polar" $\left(\mathrm{H}_{2} \mathrm{O}\right.$-dominated $)$ ice layer. In regions of higher density where almost all of the gaseous hydrogen is molecular, a relatively H-poor "nonpolar" ice layer accumulates, dominated by $\mathrm{CO}$ accreted directly from the gas. Other potential constituents such as methanol $\left(\mathrm{CH}_{3} \mathrm{OH}\right)$ and $\mathrm{CN}$-bearing species are sometimes abundant in the spectra of YSOs but have low concentrations in the 
molecular-cloud ices (e.g., Boogert et al. 2008; Gibb et al. 2004, and references therein), suggesting that photochemical or thermally driven reactions are needed for their efficient production. $\mathrm{CO}_{2}$ and $\mathrm{CH}_{3} \mathrm{OH}$ compete for the available $\mathrm{CO}$ in reaction networks based on surface chemistry: $\mathrm{CH}_{3} \mathrm{OH}$ forms by successive hydrogenation reactions in the sequence $\mathrm{CO} \rightarrow \mathrm{HCO} \rightarrow \mathrm{H}_{2} \mathrm{CO} \rightarrow \mathrm{H}_{2} \mathrm{COH} \rightarrow \mathrm{CH}_{3} \mathrm{OH}$, of which the first and third possess activation energy barriers (Cuppen et al. 2009). Whereas $\mathrm{CH}_{3} \mathrm{OH}$ can readily undergo further processing to form more complex organic species, the more stable $\mathrm{CO}_{2}$ is something of a bottleneck (Langer et al. 2000). In initiating $\mathrm{CH}_{3} \mathrm{OH}$ production, the energy released by newly born stars may thus be vital in driving chemical evolution toward greater complexity. The resulting increase in the mobility of species in the solid may also lead to physical changes such as segregation and crystallization. Ultimately, heating may result in partial or complete sublimation of the ices, and the molecules thus released may then undergo further evolution in the gas phase in hot cores or corinos around the YSO (e.g., Herbst \& van Dishoeck 2009).

The importance of the $\mathrm{CO}_{2}$ bending mode as a diagnostic signature of the composition and thermal environment of the ice mixture in which the $\mathrm{CO}_{2}$ resides was demonstrated by extensive work on laboratory analogs (Ehrenfreund et al. 1998, 1999; Dartois et al. 1999; van Broekhuizen et al. 2006; White et al. 2009). The profile shape of the $15 \mu \mathrm{m}$ absorption feature is sensitive to the molecular environment of the $\mathrm{CO}_{2}$, enabling discrimination between $\mathrm{CO}_{2}$ in polar $\left(\mathrm{H}_{2} \mathrm{O}\right.$-dominated) and nonpolar (CO-dominated) phases at low temperature, and providing evidence for formation and segregation of linked $\mathrm{CO}_{2}-\mathrm{CH}_{3} \mathrm{OH}$ molecular groups when the ices are heated. Dilute $\mathrm{CO}_{2}$-bearing amorphous ice mixtures that display smooth profiles at low temperature evolve upon heating to display subfeatures characteristic of irreversible changes as the ice begins to crystallize above 50-60 K in laboratory-based experiments (e.g., White et al. 2009). Observations with ISO and with Spitzer have demonstrated that these subfeatures are present in the spectra of many YSOs, and are (as expected) lacking in the spectra of unprocessed ices seen toward background field stars (e.g., Gerakines et al. 1999; Watson et al. 2004; Pontoppidan et al. 2008; Whittet et al. 2009).

Our group and others have developed techniques for fitting observed ice profiles with models based on laboratory spectra for interstellar analogs. In general, the observed $\mathrm{CO}_{2}$ bendingmode profile is not well matched by a single $\mathrm{CO}_{2}$-bearing ice mixture, but it can be modeled satisfactorily by a combination of two or more mixtures, of which one is $\mathrm{H}_{2} \mathrm{O}$-rich (representing the polar ice component described above) and the others are typically $\mathrm{H}_{2} \mathrm{O}$-poor. Results for unprocessed ices observed in field-star spectra are well matched by a superposition of polar and nonpolar mixtures at low temperature $(\lesssim 20 \mathrm{~K}$; Bergin et al. 2005; Whittet et al. 2007, 2009). Observed variations in the $\mathrm{CO}_{2} / \mathrm{H}_{2} \mathrm{O}$ abundance ratio between three different molecular clouds were shown to have little to no impact on the $15 \mu \mathrm{m} \mathrm{CO}_{2}$ profile, indicating that a robust benchmark has been established for comparison with regions in which the ices are exposed to harsher conditions.

The present paper extends our previous work on the field stars to encompass the rich legacy of Spitzer spectra available for low-mass YSOs (Section 2). The data are compared with the extensive library of spectra for interstellar ice analogs now available from the databases of the Astrophysics Laboratories of Leiden University (Ehrenfreund et al. 1997, 1999) and the
University of Alabama at Birmingham (UAB; White et al. 2009), spanning a wide range of temperature $(5-150 \mathrm{~K})$ as well as composition. A least-squares procedure is used (Section 3) to fit each observed profile with an appropriate combination of laboratory analogs, allowing the general properties of the ices to be characterized. The spectra are also used to determine the solid $\mathrm{CO}_{2}$ column density, $N\left(\mathrm{CO}_{2}\right)$, in each line of sight, and to investigate the correlation of $N\left(\mathrm{CO}_{2}\right)$ with extinction (Section 4$)$, with the column densities of other species in the ices, and with characteristics of the YSOs such as spectral index and the presence or absence of structure in the observed $\mathrm{CO}_{2}$ profile (Section 5). The set $\left\{\mathrm{H}_{2} \mathrm{O}, \mathrm{CO}, \mathrm{CO}_{2}\right\}$ contains the dominant quantifiable components of interstellar and preplanetary ices in most lines of sight, and we combine new and previously published results to investigate the intercorrelation of these three species with the aid of ternary plots (Section 5.3). Our conclusions are summarized in the final section.

\section{OBSERVATIONS}

\subsection{Source Selection}

Sources were chosen on the basis of known YSO status (using the SIMBAD database and references therein as a guide) with clear detections of the $15 \mu \mathrm{m} \mathrm{CO} \mathrm{CO}_{2}$ ice feature in spectra from the short-high ( $\mathrm{SH})$ module of the Spitzer Infrared Spectrometer (IRS; Houck et al. 2004). Only sources exhibiting absorption profiles deemed to have sufficient depth and signalto-noise ratio for reliable modeling were selected. The final list (Table 1) includes six sources observed as part of our Cycle 1 Spitzer program targeting the Taurus and Ophiuchus Molecular Clouds (PID 3303; Principal Investigator: D. Whittet), namely, MHO-3, IRAS 04154+2823, V410 X-ray 2, IRAS 04181+2654, ITG 25B, and Oph 125; these were supplemented by a further 22 sources in various star-forming regions, taken from the Spitzer data archive. $\mathrm{CO}_{2}$ spectra for most of these additional sources have been published previously by Pontoppidan et al. (2008). Note, however, that we adopt a somewhat different strategy for laboratory fitting compared with these authors, as detailed in Section 3.2 below.

\subsection{Data Acquisition and Reduction}

All observations reported here were obtained with the $\mathrm{SH}$ module of the Spitzer IRS, which covers the wavelength range $10-19.5 \mu \mathrm{m}$ at a resolving power $\lambda / \Delta \lambda \sim 600$ (Houck et al. 2004). Astronomical Observation Request (AOR) keys, which provide unique identification of each spectrum in the Spitzer archive, are listed in Table 1. Most of the observations were carried out in the staring mode of the IRS; however, the library of suitable spectra was extended by searching the Spitzer archive for observations of sufficient quality made in spectral mapping mode. Results for five objects in our sample are based on mapping-mode observations (L1489 IR, DF Tau, L1551 IRS5, TMC-1A, and Elias 18). Each spectrum was processed with the Spitzer Science Center pipeline version S14.0.0, which provided basic calibration data. The spectra were reduced using the $\mathrm{c} 2 \mathrm{~d}$ pipeline, which offers a reasonable estimate of the background for subtraction from sources with deep absorption features. See Pontoppidan et al. (2008) for further details of this pipeline.

\subsection{Continuum Fitting}

The flux continuum for each source was determined by loworder polynomial fitting. In addition to the $\mathrm{CO}_{2}$ feature, many 
Table 1

Summary of Observations

\begin{tabular}{|c|c|c|c|c|c|c|c|c|}
\hline Source Name & IRAS Association & Other Association & Region & $\begin{array}{l}\text { R.A. (J2000) } \\
(\mathrm{h} \mathrm{m} \mathrm{s})\end{array}$ & $\begin{array}{c}\text { Decl. (J2000) } \\
(\circ / \prime \prime)\end{array}$ & AOR Key & Date Observed & Program ID \\
\hline HH 14 & IRAS $03254+3050$ & & Lynds 1450 & 032834.51 & +310051.2 & 11827200 & 2005 Feb 9 & 172 \\
\hline B1 IRS & IRAS 03301+3057 & Pers Emb 2 & Barnard 1 & 033316.67 & +310755.0 & 15918080 & 2005 Sep 9 & 179 \\
\hline B5 IRS3 & IRAS $03439+3233$ & & Barnard 5 & 034705.81 & +324309.0 & 5635072 & 2004 Sep 29 & 172 \\
\hline B5 IRS1 & IRAS $03445+3242$ & НH366 & Barnard 5 & 034741.61 & +325143.8 & 5635328 & 2004 Feb 4 & 172 \\
\hline L1489 IR & IRAS 04016+2610 & НH360 & Taurus & 040443.07 & +261856.4 & 3528960 & 2004 Feb 8 & 2 \\
\hline MHO-3 & IRAS $04114+2757$ & & Taurus & 041430.55 & +280514.6 & 10717440 & 2005 Feb 9 & 3303 \\
\hline IRAS 04154+2823 & IRAS $04154+2823$ & & Taurus & 041832.03 & +283115.4 & 10718208 & 2004 Sep 30 & 3303 \\
\hline V410 X-ray 2 & & & Taurus & 041834.44 & +283030.2 & 10718464 & 2005 Feb 8 & 3303 \\
\hline IRAS 04181+2654 & IRAS 04181+2654 & & Taurus & 042111.46 & +270109.4 & 10719488 & 2004 Oct 4 & 3303 \\
\hline DG Tau B & & & Taurus & 042702.66 & +260530.5 & 3540992 & 2004 Feb 8 & 2 \\
\hline DF Tau & IRAS $04239+2535$ & & Taurus & 042702.80 & +254222.3 & 3530752 & 2004 Feb 8 & 2 \\
\hline L1551 IRS5 & IRAS 04287+1801 & & Lynds 1551 & 043134.08 & +180804.9 & 3531776 & 2004 Mar 5 & 2 \\
\hline TMC-1A & IRAS $04365+2535$ & & Taurus & 043935.19 & +254144.7 & 3533312 & 2004 Feb 28 & 2 \\
\hline Elias 18 & IRAS 04369+2539 & Ced 38, IC 2087 & Taurus & 043955.74 & +254502.0 & 3533312 & 2004 Feb 28 & 2 \\
\hline ITG 25B & IRAS 04370+2559 & & Taurus & 044008.00 & +260525.4 & 10722560 & 2004 Oct 4 & 3303 \\
\hline HH 47 IR & IRAS 08242-5050 & & HH 46/47 & 082543.78 & -510035.6 & 5638912 & 2003 Dec 15 & 172 \\
\hline Ced 110 IRS6 & IRAS $11057-7706$ & & Chameleon I & 110709.23 & -772304.3 & 5639680 & 2004 Sep 1 & 172 \\
\hline Cha II-28 & IRAS 12553-7651 & & Chameleon II & 125906.63 & -770740.0 & 9830912 & 2005 Mar 12 & 179 \\
\hline CG 12 & IRAS 13546-3941 & & DC $316.5+21.2$ & 135738.94 & -395600.2 & 5642752 & 2004 Jul 17 & 172 \\
\hline B228 & IRAS $15398-3359$ & & Barnard 228 & 154302.26 & -340906.7 & 5828864 & 2005 Sep 9 & 179 \\
\hline Oph 125 & & IRS 37, [GY92] 246 & Ophiuchus & 162718.17 & -242852.6 & 10727168 & 2005 Mar 18 & 3303 \\
\hline CRBR 2422.8-3423 & & & Ophiuchus & 162724.61 & -244103.3 & 9346048 & 2004 Mar 26 & 172 \\
\hline IRS 63 & IRAS 16285-2355 & & Ophiuchus & 163135.53 & -240128.3 & 12676608 & 2006 Mar 21 & 2 \\
\hline CK 1 & & EC 90 & Serpens & 182957.75 & +011405.9 & 9828352 & 2004 Sep 2 & 172 \\
\hline R CrA IRS5 & & & Corona Australis & 190148.03 & -365721.6 & 9835264 & 2005 Apr 15 & 179 \\
\hline R CrA IRS7a & & [B87] 10 & Corona Australis & 190155.32 & -365722.0 & 9835008 & 2005 Apr 15 & 179 \\
\hline L723 & IRAS 19156+1906 & & Lynds 723 & 191753.16 & +191216.6 & 14905856 & 2005 Oct 14 & 20604 \\
\hline L1262 & IRAS 23238+7401 & & Lynds 1262 & 232546.70 & +74 1737.2 & 9833728 & 2004 Oct 24 & 179 \\
\hline
\end{tabular}

sources exhibit a substantial absorption at shorter wavelengths, attributed to the wing of the broad $10 \mu \mathrm{m}$ interstellar silicate feature blended with the weaker libration mode of $\mathrm{H}_{2} \mathrm{O}$-ice. To eliminate this contribution, some spectra required separate continuum fits on each side of the $15.2 \mu \mathrm{m}$ profile. Figure 1 shows L1489IR and TMC-1A as examples of "normal" and "composite" continuum fits, respectively. In the case of L1489IR, a single fit over the entire SH spectrum, excluding the 14.7-15.9 $\mu \mathrm{m}$ segment containing the $\mathrm{CO}_{2}$ feature, was found to be satisfactory. In the case of TMC-1A, a fit to the continuum at shorter wavelengths (from 10 to $14.5 \mu \mathrm{m}$ ) was combined with a fit to the segments $14.5-14.8 \mu \mathrm{m}$ and $15.9-19.5 \mu \mathrm{m}$ at longer wavelengths. Including some of the points on the short-wavelength side of the $\mathrm{CO}_{2}$ feature allowed us to create a smooth continuum transition over the absorption band. The optical depth spectrum was then calculated routinely for each source from the ratio of the continuum to the observed flux, i.e., $\tau(\lambda)=\ln \left[F_{\text {cont }}(\lambda) / F_{\text {obs }}(\lambda)\right]$. The contribution of uncertainties in continuum fits to errors in column density estimates (Section 5) are small (typically a few percent) and no greater than the statistical errors in the data in each case.

\section{FITTING THE $\mathrm{CO}_{2}$ PROFILE}

\subsection{Laboratory Databases}

Astrochemical laboratories are a necessary and influential resource for infrared astronomy. As noted in Section 1, spectra for a wide range of specific ice mixtures are available for use in fitting astronomical spectra obtained from infrared observations. To fit the Spitzer $\mathrm{CO}_{2}$ spectra, we utilized the databases available

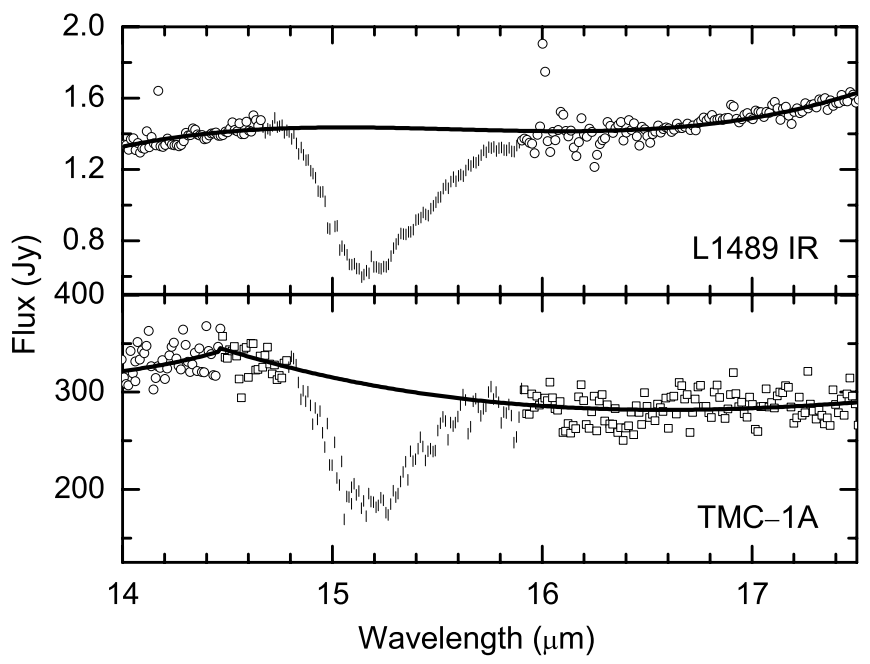

Figure 1. Examples of local continuum fitting. Vertical hatches are data points within the absorption profiles not included in the fits. L1489IR (top panel) illustrates the fitting method used for the majority of our spectra, where only a single polynomial fit to the continuum data points (represented by circles) was required. The fit to TMC-1A (bottom panel) utilizes two intersecting polynomials, one fit to the circles and one to the squares. See the text (Section 2.3) for details.

from the Astrophysical Ice Laboratory at Leiden University ${ }^{6}$ and the Astrophysics Laboratory at the UAB. ${ }^{7}$ These groups have created and characterized ice mixtures with a range of compositions and exposed to a range of temperatures, resulting

\footnotetext{
6 Ehrenfreund et al. (1997, 1999); see http://www.strw.leidenuniv.nl/ lab.

7 White et al. (2009); see http://www.phy.uab.edu/labastro/databases.html.
} 
in libraries that contain a total of 1381 spectra relevant to this project, all of which are public or available upon request. The fitting routine draws fit components from this suite of spectra, as described below (Section 3.2).

Realistic simulations of interstellar spectra calculated from laboratory spectra must take particle size and shape effects into consideration. These effects have been found to have significant impact on the structure of strong absorption features at shorter infrared wavelengths (e.g., Tielens et al. 1991; Ehrenfreund et al. 1997), including the $4.1 \mu \mathrm{m}$ stretching-mode feature of $\mathrm{CO}_{2}$ (e.g., Gerakines et al. 1999). At the wavelength of the $\mathrm{CO}_{2}$ bending mode, however, the grains are most likely well into the Rayleigh limit, i.e., $2 \pi a \ll 15 \mu \mathrm{m}$, where $a$ is the radius of the largest grains (Pontoppidan et al. 2008): in this situation, grain size and shape effects typically have negligible influence on profile shape. They are also less important for the relatively broad ice features seen in polar mixtures, in comparison to the sharply peaked features of pure $\mathrm{CO}_{2}$ ices (Ehrenfreund et al. 1997 and references therein; note that pure $\mathrm{CO}_{2}$ ices were not used in any of our fits). Moreover, optical constants used to calculate grain size and shape corrections are controversial (e.g., Boogert 1999; Moore et al. 2010) and can introduce widely varying results according to assumptions made. In view of these considerations, we have chosen to omit grain shape corrections from the laboratory spectra used in the current work. All of our astronomical spectra are well fit by uncorrected laboratory data, and the fits are interpreted primarily as a qualitative description of the ice components toward different lines of sight.

\subsection{Fitting Procedure}

The Spitzer optical depth spectra were input to a fitting routine developed by Gerakines et al. (1999), similar to that used by Chiar et al. (1995). The routine determines the best fit to each spectrum via $\chi_{v}^{2}$ (reduced chi-squared) minimization. The fitting procedure assumes that the profile can be modeled by a combination of two distinct ice components, as in our previous work: this choice is justified as the smallest number of components capable of giving an acceptable fit, but it should be noted that deconvolution into as many as five components has been used to fit $\mathrm{CO}_{2}$ bending-mode profiles in some previous work. ${ }^{8}$ Uniqueness is naturally an important issue, and we adopt the strategy that is preferable to use only as many components as will make a good fit. In reality, most fitting routines do not provide "unique" fits; often, several combinations of ices will provide acceptable fits (with similar $\chi_{v}^{2}$ residuals) to a given observed spectrum. Our aim is to obtain a "family" of fits that are similar in character (i.e., with comparable ratios of polar to nonpolar ices or crystalline ices at similar temperatures), with as few components as deemed necessary, based on observation. This procedure enables the general nature of the ices in a given line of sight to be characterized.

The ices in the laboratory database were divided into four groups: (1) 21 polar ices $^{9}$ at temperatures below $60 \mathrm{~K}$, (2) 33 nonpolar ices at temperatures below $30 \mathrm{~K}$, (3) 22 polar ices with no methanol constituent at temperatures above $60 \mathrm{~K}$, and

\footnotetext{
8 For example, Pontoppidan et al. (2008) report excellent fits to some 40 spectra in the $\mathrm{c} 2 \mathrm{~d}$ sample utilizing a superposition of five individual ice components derived from laboratory data and allowed to vary in amplitude from source to source; our fitting strategy differs from theirs in that we allow a suite of possible laboratory spectra for each of two components.

9 We rejected any "polar" ice mixture containing more than $33 \% \mathrm{CO}_{2}$ relative to $\mathrm{H}_{2} \mathrm{O}$ on the basis of existing observational constraints (e.g., Whittet et al. 2007, 2009; Knez et al. 2005; Zasowski et al. 2009).
}

(4) 954 ices containing various mixtures of $\mathrm{H}_{2} \mathrm{O}, \mathrm{CO}_{2}$, and $\mathrm{CH}_{3} \mathrm{OH}$ at temperatures ranging from 5 to $150 \mathrm{~K}$. The key difference between ices in groups (1) and (3) is the occurrence of crystallization features in the latter, which first appear at $\sim 60 \mathrm{~K}$. Each fit is calculated by combining spectra from two of these groups:

$$
\tau(\lambda)=a_{1} \tau_{\mathrm{lab} 1}(\lambda)+a_{2} \tau_{\mathrm{lab} 2}(\lambda)
$$

where $\tau_{\mathrm{lab} 1}(\lambda)$ and $\tau_{\mathrm{lab} 2}(\lambda)$ are the normalized optical depth spectra of the two laboratory ice components in the fit, and the scale factors $a_{1}$ and $a_{2}$ are free parameters. Component 1 is assumed to be a polar ice from group (1) in every case; component 2 is selected from the other groups, the final choice being determined by $\chi_{v}^{2}$ minimization.

\subsection{Results}

Our laboratory fits, shown in Figures 2 and 3 and summarized in Table 2, are separated into two main evolutionary groups: (1) polar + nonpolar ("P+NP") and (2) polar + crystallized ice mixture ("P+CIM"). Recall that all fits within these two evolutionary groups were accomplished with the constraint of using only two ice components for each fit. See Table 2 for detailed fit information.

Spectra of YSOs in the P+NP group (Figure 2) exhibit $\mathrm{CO}_{2}$ profiles that are best fit by a mix of polar $\mathrm{CO}_{2}$ ice (i.e., $\mathrm{CO}_{2}$ embedded in an $\mathrm{H}_{2} \mathrm{O}$ matrix) and nonpolar $\mathrm{CO}_{2}$ ice (i.e., $\mathrm{CO}_{2}$ embedded in a $\mathrm{CO}$ matrix), all at temperatures $\lesssim 42 \mathrm{~K}$. Some of the nonpolar laboratory ice spectra include $\mathrm{O}_{2}$, but $\mathrm{CO}_{2}$ or $\mathrm{CO}$ is always the dominant constituent. The observed profiles and resulting fits for objects in this group closely resemble those of background field stars such as Elias 16, CK2 and Q21-1 (Bergin et al. 2005; Whittet et al. 2009). We deduce that the lines of sight to the P+NP YSOs are sampling regions where the ices are thermally unevolved. This could arise because (1) absorption in the line of sight is dominated by cold foreground material in the molecular cloud and (2) material local to the YSO in the line of sight has not (yet) been heated significantly, or a combination of (1) and (2).

Spectra of YSOs in the P+CIM evolutionary group exhibit double-peaked structure in the $15 \mu \mathrm{m}$ profile characteristic of crystallized $\mathrm{CO}_{2}$ ices (Figure 3). Best fits combine polar and crystallized ice mixtures at temperatures $5-40 \mathrm{~K}$ and $\gtrsim 100 \mathrm{~K}$, respectively. The profiles typically also display a shoulder at the long wavelength side of the feature attributed to complexing of $\mathrm{CH}_{3} \mathrm{OH}$ with $\mathrm{CO}_{2}$. As $\mathrm{CH}_{3} \mathrm{OH}$ makes only a minor contribution to the total absorption in the feature, and the CIM ice component may not contain all the $\mathrm{CH}_{3} \mathrm{OH}$ in a given line of sight, our fits are not expected to provide precise estimates of $\mathrm{CH}_{3} \mathrm{OH}$ abundance toward individual sources. A range of 1\%-15\% with respect to $\mathrm{H}_{2} \mathrm{O}$ is implied by our results (Table 2), consistent with that determined from observations at other wavelengths (e.g., Boogert et al. 2008). Evidence of crystallization suggests that ices toward these sources have been significantly heated, most probably as the result of exposure to infrared radiation from the embedded YSO, consistent with the assumption (Section 1) that an energy source is needed to drive efficient $\mathrm{CH}_{3} \mathrm{OH}$ production.

\section{EXTINCTION}

In this section, we use near-infrared $(J H K)$ photometry to estimate interstellar reddening and extinction toward sources in our sample. All available photometry is listed in Table 3 and is taken from the Two Micron All Sky Survey (2MASS; 

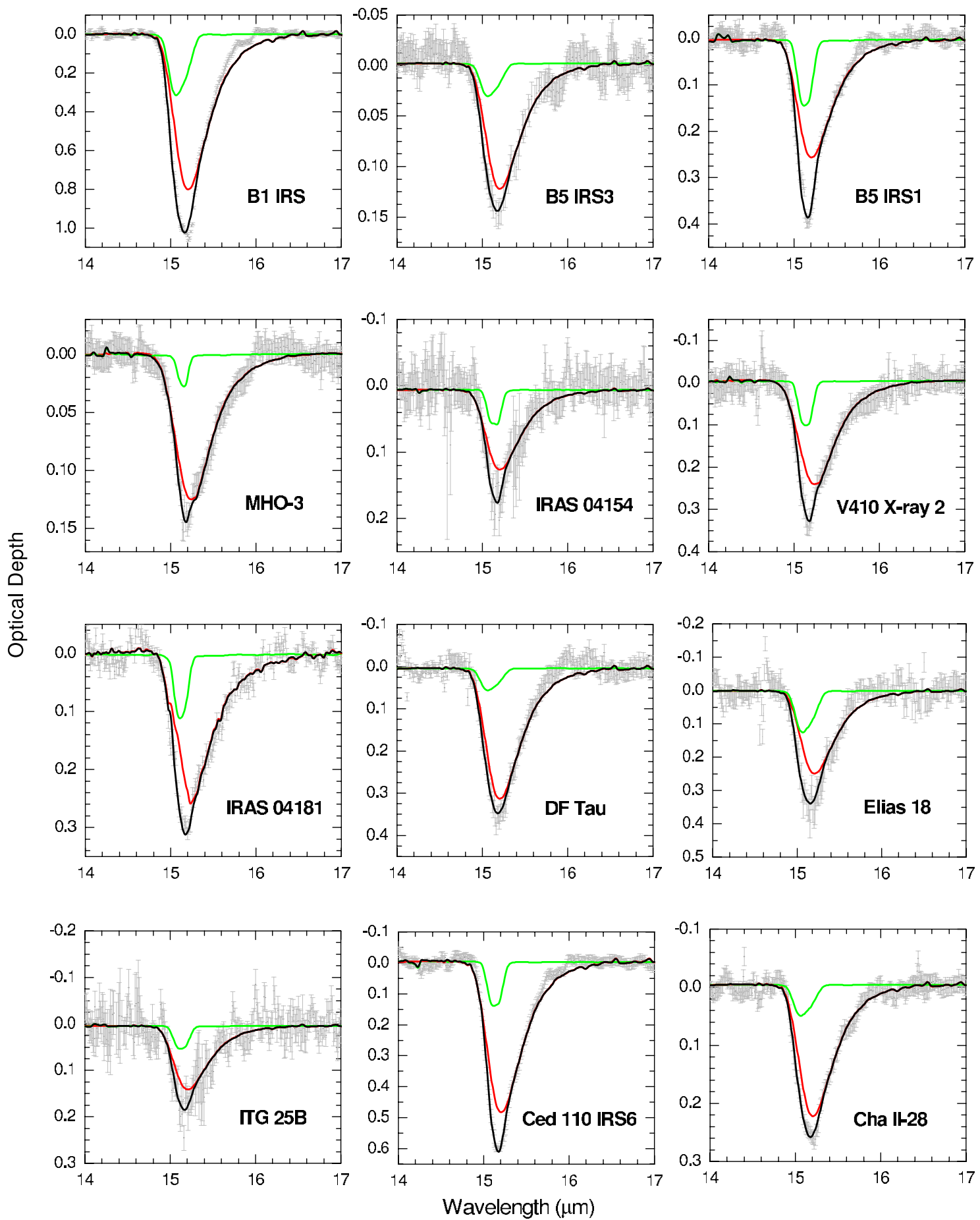

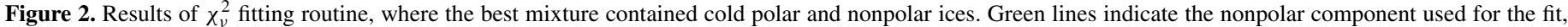
red lines indicate the polar component, and black lines are the sum of the two components.

(A color version of this figure is available in the online journal.)

Skrutskie et al. 2006) unless otherwise indicated in the notes. The $J-H, H-K$ color-color diagram is shown in Figure 4 for the subset of 15 sources that have reliable photometry available in all three bands; reddened background field stars in the Taurus region (Whittet et al. 2007) are also plotted for comparison. In order to interpret the distribution of data in Figure 4 in terms of reddening, and convert to total extinction, an extinction law must be assumed, i.e., a form for the wavelength dependence of extinction. This is expected to vary according to the physical properties of the dust in a given environment, but the dispersion tends to be small in the infrared (e.g., Martin \& Whittet 1990). We assume that the mean extinction law for dust in the Taurus dark cloud (Whittet et al. 2001, 2007) is applicable to our sample. The pertinent relationships describing this law are the slope of the reddening vector in the $J-H, H-K$ diagram:

$$
E_{J-H}=1.75 E_{H-K},
$$

where $E$ represents color excess (i.e., reddening), and the conversion from reddening in the infrared to total (visual) extinction: 

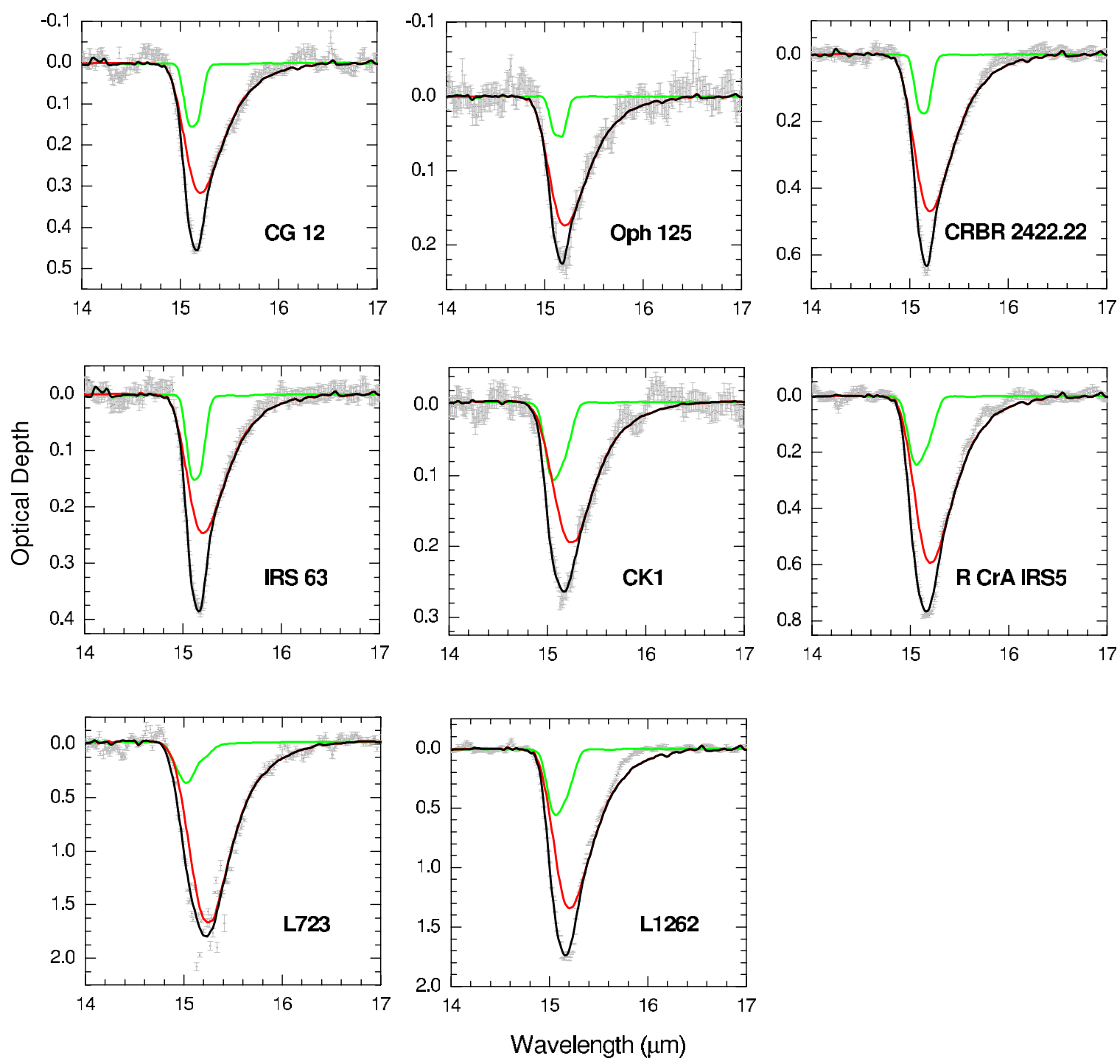

Figure 2. (Continued)

$$
A_{V}=5.2 E_{J-K}=5.2\left(E_{J-H}+E_{H-K}\right) .
$$

The solid diagonal lines in Figure 4 have a slope of 1.75 (Equation (2)) and denote the upper and lower bounds of the region where normal reddened stars are expected to reside. A few YSOs in our sample plot with the field stars in this region, but the majority lie below and to the right, in the zone between the lower solid line and the dashed line. This is expected in cases where infrared emission from heated circumstellar dust is present, introducing additional flux at the longer wavelengths. An excess in $H-K$ implies that dust close to the YSO reaches temperatures $\sim 1000 \mathrm{~K}$, i.e., well above those responsible for sublimation and crystallization of ices; thus, a one-to-one correspondence between $H-K$ excess and evidence of thermal processing in the ice spectra is not expected. We note that 2 out of $2 \mathrm{P}+\mathrm{CIM}$ sources and 9 out of $13 \mathrm{P}+\mathrm{NP}$ sources show evidence of significant $H-K$ excess in Figure 4. In the latter case, the lack of evidence for annealing suggests that most of the ice toward these YSOs is in cold molecular cloud material remote from the source rather than the warm circumstellar disk or envelope: warm dust close to the source may contribute only a small fraction of the total opacity.
For YSOs occupying the field-stars region in Figure 4, estimation of the extinction is straightforward, following the method used by Shenoy et al. (2008): in each case the displacement required to deredden the star onto intrinsic color lines provide measures of $E_{J-H}$ and $E_{H-K}$ in Equation (3). The intrinsic colors of the YSOs are generally unknown or poorly constrained and the range of possible displacements results in errors of \pm 2 mag in the estimated values of $A_{V}$ (Table 3 ). For YSOs in the circumstellar excess region, we assume that contamination affects only $H-K$ and not $J-H$ (reasonable for dust temperatures $\lesssim 1000 \mathrm{~K}$ ) and estimate $A_{V}=8.2 E_{J-H}$ (Equations (2) and (3)), assuming a mean intrinsic color $(J-H)_{0}=0.6$.

\section{COLUMN DENSITIES AND CORRELATIONS}

Using our optical depth spectra, column densities were calculated from the relation

$$
N\left(\mathrm{CO}_{2}\right)=\frac{1}{A} \int \tau d \nu,
$$

where $\tau$ is the optical depth, $v\left(\mathrm{~cm}^{-1}\right)$ is the wavenumber, and $A$ $\left(\mathrm{cm}\right.$ molecule $\left.{ }^{-1}\right)$ is the band strength of the $\mathrm{CO}_{2}$ absorption 

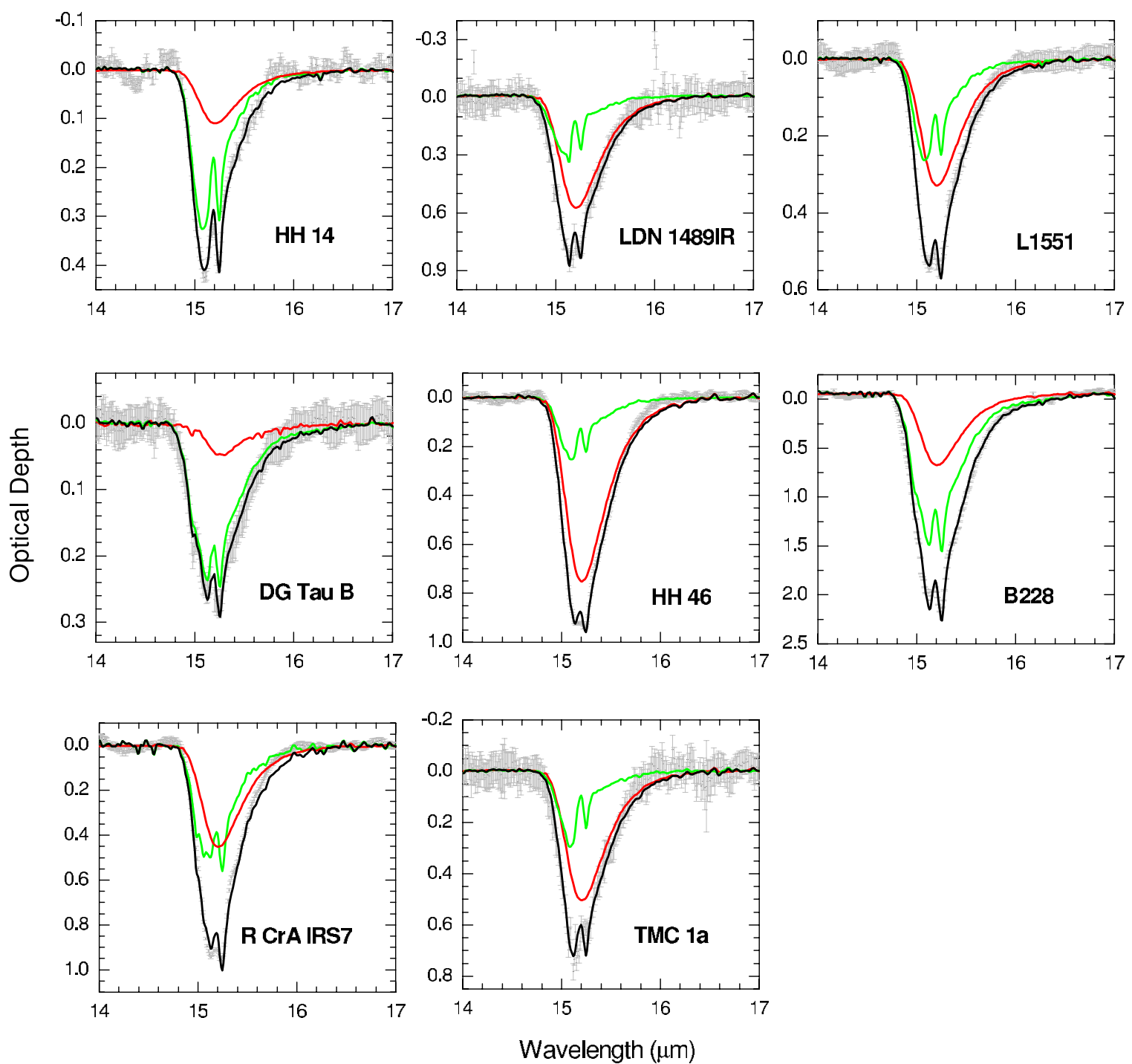

Figure 3. Results of the $\chi_{v}^{2}$ fitting routine, where the best mixture contained cold polar ice and a crystallized ice mixture (CIM). Green lines indicate the CIM component, red lines indicate the polar component, and black lines are the sum of the two components.

(A color version of this figure is available in the online journal.)

feature. Band strengths are measured in the laboratory to accuracies of typically a few percent. Gerakines et al. (1995) showed that the band strength of pure solid $\mathrm{CO}_{2}$ at $15 \mu \mathrm{m}$ is not significantly different from that of $\mathrm{CO}_{2}$ diluted in an $\mathrm{H}_{2} \mathrm{O}$ matrix, i.e., the results agree to within the experimental uncertainties. We therefore adopt the pure $\mathrm{CO}_{2}$ band strength $\left(1.1 \times 10^{-17} \mathrm{~cm}\right.$ molecule $\left.{ }^{-1}\right)$ for consistency with most previous work (Gerakines et al. 1999; Nummelin et al. 2001; Pontoppidan et al. 2008; Whittet et al. 2007, 2009). Note that column densities from Zasowski et al. (2009) should be scaled up by a factor of 1.36 (i.e., 1.5/1.1) for comparison with our results and those from the papers listed above, to allow for the different band strength assumed by these authors.

Each optical depth spectrum (Figures 2 and 3 ) was integrated between bounds chosen on a case-by-case basis. This was done to ensure that the entire solid $\mathrm{CO}_{2}$ feature was always included, notwithstanding small source-to-source variations in the position and width of the feature. The typical wavelength range of the integration was $14.8-16.3 \mu \mathrm{m}$. Table 3 lists column density results for both total $\mathrm{CO}_{2}$ and the polar, nonpolar, and crystallized $\mathrm{CO}_{2}$ components arising from the profile fitting
(Section 3), along with literature values for $\mathrm{H}_{2} \mathrm{O}$ and $\mathrm{CO}$. Uncertainties in our results take into account both continuum fitting uncertainties (Section 2.3) and noise in the observational data.

\subsection{Correlation with Extinction}

Extinction $\left(A_{V}\right)$ effectively measures the mean dust column density in a given line of sight. Figure 5 plots total $N\left(\mathrm{CO}_{2}\right)$ versus $A_{V}$, as available, for YSOs in our sample (Table 3). Also shown for comparison are data for background field stars toward three clouds. Field stars located behind the best sampled cloud (Taurus) yield a strong linear correlation (correlation coefficient $R=0.97$ ), the intercept of which indicates an extinction threshold, i.e., the minimum extinction for solid $\mathrm{CO}_{2}$ detection, of $4.3 \pm 1.0 \mathrm{mag}$ (Whittet et al. 2007). Data for field stars behind other clouds are currently limited to just two lines of sight, CK2 in Serpens and Q21-1 in IC5146 (Whittet et al. 2009). It is notable that the distribution of YSOs in Figure 5 also suggests a linear trend, if three outliers are disregarded, but with a $\mathrm{CO}_{2}$ abundance per unit extinction that is systematically 
Table 2

Summary of Laboratory Fits

\begin{tabular}{|c|c|c|c|c|c|c|c|c|}
\hline Fit Group & Source Name & Polar Ice & $T(\mathrm{~K})$ & $\tau$ & Nonpolar/CIM Ice & $T(\mathrm{~K})$ & $\tau$ & $\chi_{v}^{2}$ \\
\hline \multirow[t]{20}{*}{ Polar + Nonpolar } & B1 IRS & $\mathrm{H}_{2} \mathrm{O}: \mathrm{CO}_{2}: \mathrm{CO}=100: 20: 3$ & 20 & 0.802 & $\mathrm{CO}: \mathrm{CO}_{2}=100: 70$ & 10 & 0.316 & 28.6 \\
\hline & B5 IRS3 & $\mathrm{H}_{2} \mathrm{O}: \mathrm{CO}_{2}: \mathrm{CO}=100: 20: 3$ & 20 & 0.124 & $\mathrm{CO}: \mathrm{CO}_{2}=100: 23$ & 10 & 0.032 & 0.24 \\
\hline & B5 IRS1 & $\mathrm{H}_{2} \mathrm{O}: \mathrm{CO}_{2}: \mathrm{CO}=100: 20: 3$ & 20 & 0.254 & $\mathrm{CO}: \mathrm{CO}_{2}=100: 26$ & 10 & 0.142 & 2.64 \\
\hline & MHO-3 & $\mathrm{H}_{2} \mathrm{O}: \mathrm{CO}_{2}=100: 14$ & 10 & 0.125 & $\mathrm{CO}: \mathrm{O}_{2}: \mathrm{CO}_{2}=10: 20: 11$ & 10 & 0.028 & 0.39 \\
\hline & IRAS $04154+2823$ & $\mathrm{H}_{2} \mathrm{O}: \mathrm{CO}_{2}: \mathrm{CO}=100: 20: 3$ & 20 & 0.120 & $\mathrm{CO}: \mathrm{O}_{2}: \mathrm{CO}_{2}=100: 50: 16$ & 10 & 0.052 & 0.54 \\
\hline & V410 X-ray 2 & $\mathrm{H}_{2} \mathrm{O}: \mathrm{CO}_{2}=100: 14$ & 10 & 0.244 & $\mathrm{CO}: \mathrm{O}_{2}: \mathrm{CO}_{2}=100: 11: 20$ & 10 & 0.105 & 0.19 \\
\hline & IRAS $04181+2654$ & $\mathrm{H}_{2} \mathrm{O}: \mathrm{CO}_{2}=5: 1$ & 30 & 0.257 & $\mathrm{CO}: \mathrm{CO}_{2}=100: 23$ & 30 & 0.110 & 2.39 \\
\hline & DF Tau & $\mathrm{H}_{2} \mathrm{O}: \mathrm{CO}_{2}: \mathrm{CO}=100: 20: 3$ & 20 & 0.301 & $\mathrm{CO}: \mathrm{CO}_{2}=100: 70$ & 10 & 0.052 & 1.18 \\
\hline & Elias 18 & $\mathrm{H}_{2} \mathrm{O}: \mathrm{CO}_{2}: \mathrm{CO}=100: 20: 3$ & 20 & 0.504 & $\mathrm{H}_{2} \mathrm{O}: \mathrm{CH}_{3} \mathrm{OH}: \mathrm{CO}_{2}=1: 1.5: 1$ & 110 & 0.303 & 1.03 \\
\hline & ITG 25B & $\mathrm{H}_{2} \mathrm{O}: \mathrm{CO}_{2}: \mathrm{CO}=100: 20: 3$ & 20 & 0.137 & $\mathrm{CO}: \mathrm{CO}_{2}=100: 26$ & 10 & 0.049 & 0.97 \\
\hline & Ced 110 IRS6 & $\mathrm{H}_{2} \mathrm{O}: \mathrm{CO}_{2}: \mathrm{CO}=100: 20: 3$ & 20 & 0.485 & $\mathrm{CO}: \mathrm{O}_{2}: \mathrm{CO}_{2}=100: 10: 23$ & 10 & 0.142 & 3.03 \\
\hline & Cha II-28 & $\mathrm{H}_{2} \mathrm{O}: \mathrm{CO}_{2}: \mathrm{CO}=100: 20: 3$ & 20 & 0.226 & $\mathrm{CO}: \mathrm{CO}_{2}=100: 70$ & 10 & 0.053 & 0.64 \\
\hline & CG 12 & $\mathrm{H}_{2} \mathrm{O}: \mathrm{CO}_{2}: \mathrm{CO}=100: 20: 3$ & 20 & 0.315 & $\mathrm{CO}: \mathrm{CO}_{2}=100: 26$ & 10 & 0.155 & 2.24 \\
\hline & Oph 125 & $\mathrm{H}_{2} \mathrm{O}: \mathrm{CO}_{2}: \mathrm{CO}=100: 20: 3$ & 20 & 0.174 & $\mathrm{CO}: \mathrm{O}_{2}: \mathrm{CO}_{2}=100: 50: 16$ & 10 & 0.054 & 0.94 \\
\hline & CRBR 2422.8-3423 & $\mathrm{H}_{2} \mathrm{O}: \mathrm{CO}_{2}: \mathrm{CO}=100: 20: 3$ & 20 & 0.468 & $\mathrm{CO}: \mathrm{O}_{2}: \mathrm{CO}_{2}=100: 11: 20$ & 10 & 0.176 & 2.85 \\
\hline & IRS 63 & $\mathrm{H}_{2} \mathrm{O}: \mathrm{CO}_{2}: \mathrm{CO}=100: 20: 3$ & 20 & 0.246 & $\mathrm{CO}: \mathrm{CO}_{2}=100: 26$ & 10 & 0.152 & 1.61 \\
\hline & CK 1 & $\mathrm{H}_{2} \mathrm{O}: \mathrm{CO}_{2}=100: 14$ & 10 & 0.198 & $\mathrm{CO}: \mathrm{CO}_{2}=100: 70$ & 10 & 0.110 & 0.79 \\
\hline & R CrA IRS5 & $\mathrm{H}_{2} \mathrm{O}: \mathrm{CO}_{2}: \mathrm{CO}=100: 20: 3$ & 20 & 0.592 & $\mathrm{CO}: \mathrm{CO}_{2}=100: 70$ & 10 & 0.243 & 8.56 \\
\hline & L723 & $\mathrm{H}_{2} \mathrm{O}: \mathrm{CO}_{2}=100: 14$ & 10 & 1.684 & $\mathrm{H}_{2} \mathrm{O}: \mathrm{CO}_{2}=1: 6$ & 42 & 0.379 & 34.4 \\
\hline & L1262 & $\mathrm{H}_{2} \mathrm{O}: \mathrm{CO}_{2}: \mathrm{CO}=100: 20: 3$ & 20 & 1.340 & $\mathrm{CO}: \mathrm{CO}_{2}=100: 70$ & 10 & 0.557 & 150.0 \\
\hline \multirow[t]{8}{*}{ Polar + CIM } & HH 14 & $\mathrm{H}_{2} \mathrm{O}: \mathrm{CO}_{2}: \mathrm{CO}=100: 20: 3$ & 20 & 0.107 & $\mathrm{H}_{2} \mathrm{O}: \mathrm{CH}_{3} \mathrm{OH}: \mathrm{H}_{2} \mathrm{O}=2: 0.5: 1$ & 135 & 0.324 & 2.91 \\
\hline & L1489 IR & $\mathrm{H}_{2} \mathrm{O}: \mathrm{CO}_{2}: \mathrm{CO}=100: 20: 3$ & 20 & 0.583 & $\mathrm{H}_{2} \mathrm{O}: \mathrm{CH}_{3} \mathrm{OH}: \mathrm{CO}_{2}=1: 1: 1$ & 115 & 0.344 & 0.90 \\
\hline & DG Tau B & $\mathrm{H}_{2} \mathrm{O}: \mathrm{CO}_{2}=10: 1$ & 40 & 0.047 & $\mathrm{H}_{2} \mathrm{O}: \mathrm{CH}_{3} \mathrm{OH}: \mathrm{CO}_{2}=2: 0.5: 1$ & 125 & 0.245 & 0.25 \\
\hline & L1551 IRS5 & $\mathrm{H}_{2} \mathrm{O}: \mathrm{CO}_{2}: \mathrm{CO}=100: 20: 3$ & 20 & 0.328 & $\mathrm{H}_{2} \mathrm{O}: \mathrm{CH}_{3} \mathrm{OH}: \mathrm{CO}_{2}=2: 0.5: 1$ & 110 & 0.262 & 0.89 \\
\hline & TMC-1A & $\mathrm{H}_{2} \mathrm{O}: \mathrm{CO}_{2}: \mathrm{CO}=100: 20: 3$ & 20 & 0.506 & $\mathrm{H}_{2} \mathrm{O}: \mathrm{CH}_{3} \mathrm{OH}: \mathrm{CO}_{2}=2: 1.5: 1$ & 110 & 0.297 & 1.04 \\
\hline & HH 47 IR & $\mathrm{H}_{2} \mathrm{O}: \mathrm{CO}_{2}: \mathrm{CO}=100: 20: 3$ & 20 & 0.751 & $\mathrm{H}_{2} \mathrm{O}: \mathrm{CH}_{3} \mathrm{OH}: \mathrm{CO}_{2}=2: 1: 1$ & 125 & 0.253 & 8.00 \\
\hline & B228 & $\mathrm{H}_{2} \mathrm{O}: \mathrm{CO}_{2}: \mathrm{CO}=100: 20: 3$ & 20 & 0.728 & $\mathrm{H}_{2} \mathrm{O}: \mathrm{CH}_{3} \mathrm{OH}: \mathrm{CO}_{2}=2: 0.5: 1$ & 125 & 1.611 & 110.0 \\
\hline & R CrA IRS7a & $\mathrm{H}_{2} \mathrm{O}: \mathrm{CO}_{2}: \mathrm{CO}=100: 20: 3$ & 20 & 0.451 & $\mathrm{H}_{2} \mathrm{O}: \mathrm{CH}_{3} \mathrm{OH}: \mathrm{CO}_{2}=2: 0.1: 1$ & 140 & 0.559 & 19.1 \\
\hline
\end{tabular}

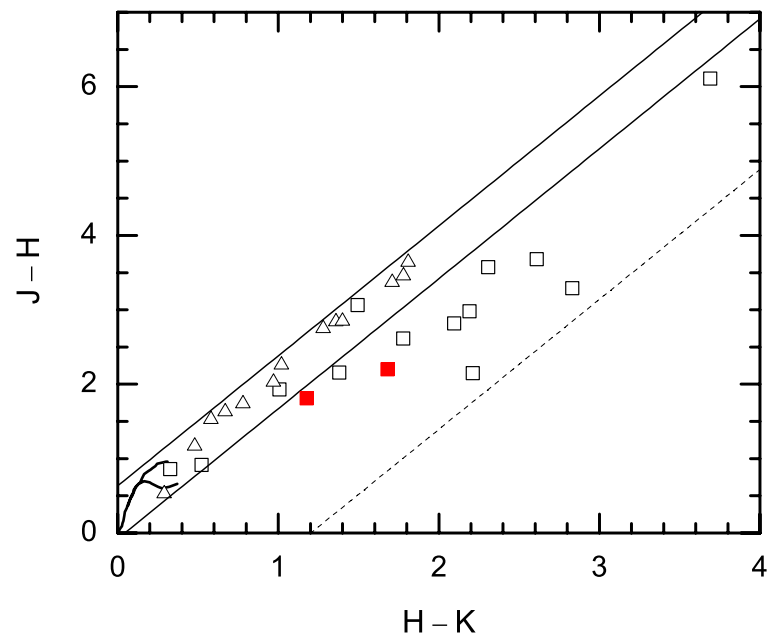

Figure 4. $J H K$ color-color diagram for YSOs from this paper and Taurus field stars from Whittet et al. (2007). Triangles denote field stars, open squares denote $\mathrm{P}+\mathrm{NP}$ YSOs, and filled (red) squares denote P+CIM YSOs. Photometry for Ced 110 IRS6 is from Persi et al. (2001); all other photometry is from the 2MASS database (Table 3). Solid curves near the origin are intrinsic colors, indicating the expected loci of normal unreddened stars of luminosity classes III and V. Normal reddened stars are expected to lie between the solid diagonal lines for an extinction law $E_{J-H}=1.75 E_{H-K}$ (Whittet et al. 2007). YSOs with significant infrared excess emission at $2 \mu \mathrm{m}$ are expected to reside in the zone between the lower solid line and the dashed line.

(A color version of this figure is available in the online journal.)

$\sim 30 \%$ higher. All YSOs that follow this general trend have $\mathrm{P}+\mathrm{NP}$ classification from our fitting procedure, i.e., their $\mathrm{CO}_{2}$ profiles resemble those of field stars. It should also be noted that two "non-Taurus" field stars (CK2 and Q21-1) plot with the
YSOs, having significantly more $\mathrm{CO}_{2}$ per unit extinction than expected based on the Taurus correlation. One interpretation is that the Taurus cloud is not prototypical (see Whittet et al. 2009 for further discussion) and that $\mathrm{CO}_{2}$ abundance per unit dust column exhibited by $\mathrm{CK} 2$ and Q21-1 is more representative of ice chemistry: in this case, the $\mathrm{CO}_{2}$ abundance found in the $\mathrm{P}+\mathrm{NP}$ YSOs may be attributed entirely ices in the foreground with no local enhancement. However, considering data for the Taurus cloud alone (solid black symbols), we see a significant difference in the trends for field stars and YSOs. This suggests the possibility of local $\mathrm{CO}_{2}$ production toward the YSOs.

We next consider the outliers displaying large apparent $\mathrm{CO}_{2}$ enhancements relative to $A_{V}$ in Figure 5. Two out of the three in this group (L1551 IRS5 and HH14) are P+CIM sources, and it seems plausible that $\mathrm{CO}_{2}$ production is driven by a local radiation field in these lines of sight that is also responsible for crystallization of the ices. However, an alternative (and less interesting) explanation could arise if the observed values of $A_{V}$ and $N\left(\mathrm{CO}_{2}\right)$, which are estimated from near- and mid-infrared data, respectively, do not sample the exact same column of dust. This latter explanation seems particularly likely in the case of DF Tau, which is expected to have negligible $\mathrm{CO}_{2}$ abundance on the basis of its very low apparent $A_{V}$. DF Tau is a binary system in which one component is undergoing flare activity (Li et al. 2001; Lamzin et al. 2001). If the dust distribution is uneven, it may be the case that the star with the lowest extinction dominates the near-infrared flux and the star with the highest extinction dominates the flux at $15 \mu \mathrm{m}$.

\subsection{Correlation with Other Ices}

We next consider the intercorrelation of ice column densities in our sample. Figures 6 and 7 plot available $N\left(\mathrm{H}_{2} \mathrm{O}\right)$ versus 
Table 3

Magnitudes, Extinctions, and Column Densities ${ }^{\mathrm{a}}$

\begin{tabular}{|c|c|c|c|c|c|c|c|c|c|c|c|c|}
\hline Source & $J$ & $H$ & $K$ & $A_{V}$ & $\alpha$ & $N\left(\mathrm{CO}_{2}\right)$ & $N\left(\mathrm{H}_{2} \mathrm{O}\right)$ & $N(\mathrm{CO})$ & $\begin{array}{c}N\left(\mathrm{CO}_{2}\right) \\
\text { Polar }\end{array}$ & $\begin{array}{l}N\left(\mathrm{CO}_{2}\right) \\
\text { Nonpolar }\end{array}$ & $\begin{array}{c}N\left(\mathrm{CO}_{2}\right) \\
\text { Crystallized }\end{array}$ & Notes $^{\mathrm{b}}$ \\
\hline HH 14 & 15.00 & 13.19 & 12.01 & 11.0 & 1.09 & $9.48 \pm 0.55$ & $40.5 \pm 3.7$ & $3.1 \pm 1.6$ & 3.01 & $\ldots$ & 6.46 & 1 \\
\hline B1 IRS & 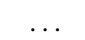 & & 14.21 & . & 1.81 & $24.1 \pm 0.2$ & $104 \pm 23$ & & 20.11 & 4.01 & $\ldots$ & 1 \\
\hline B5 IRS3 & 16.41 & 14.26 & 12.05 & 13.0 & 0.75 & $3.23 \pm 0.25$ & $10.1 \pm 0.9$ & $2.9 \pm 1.3$ & 2.82 & 0.41 & $\ldots$ & 1 \\
\hline B5 IRS1 & 17.34 & 14.05 & 11.21 & 23.0 & 1.22 & $8.34 \pm 0.20$ & $22.6 \pm 2.8$ & $11.5 \pm 1.9$ & 6.89 & 1.44 & $\ldots$ & 1 \\
\hline L1489 IR & $\ldots$ & & $\ldots$ & $\ldots$ & $\ldots$ & $19.3 \pm 1.7$ & $47 \pm 2.8$ & $9.0 \pm 0.5$ & 13.29 & $\ldots$ & 5.98 & 1 \\
\hline MHO-3 & 11.18 & 9.25 & 8.24 & 11.0 & $\ldots$ & $3.57 \pm 0.33$ & $\ldots$ & $\ldots$ & 3.32 & 0.25 & $\ldots$ & \\
\hline IRAS $04154+2823$ & 15.19 & 12.37 & 10.27 & 19.0 & 0.21 & $4.47 \pm 1.27$ & $8.75 \pm 0.8$ & $\ldots$ & 4.02 & 0.45 & $\ldots$ & 2 \\
\hline V410 X-ray 2 & 13.77 & 10.71 & 9.22 & 19.6 & -0.18 & $6.85 \pm 0.24$ & $\ldots$ & $\ldots$ & 5.86 & 0.98 & $\ldots$ & \\
\hline IRAS $04181+2654$ & 16.22 & 12.65 & 10.34 & 25.0 & 0.30 & $7.67 \pm 0.24$ & $25 \pm 2$ & $7.7 \pm 0.8$ & 6.31 & 1.35 & $\ldots$ & 2 \\
\hline DG Tau B & 15.60 & $\ldots$ & $\ldots$ & $\ldots$ & $\ldots$ & $7.49 \pm 0.74$ & $26.3 \pm 2.6$ & $2.8 \pm 1.4$ & 1.42 & $\ldots$ & 6.06 & 1 \\
\hline DF Tau & 8.17 & 7.26 & 6.73 & 3.0 & -1.04 & $9.22 \pm 0.65$ & $\ldots$ & $\ldots$ & 8.54 & 0.68 & $\ldots$ & \\
\hline L1551 IRS5 & 13.71 & 11.51 & 9.82 & 14.0 & 1.93 & $13.70 \pm 0.61$ & $\ldots$ & $\ldots$ & 8.39 & $\ldots$ & 5.31 & \\
\hline TMC-1A & $\ldots$ & 13.752 & 10.84 & $\ldots$ & 1.29 & $17.52 \pm 0.62$ & $\ldots$ & $\ldots$ & 12.41 & $\ldots$ & 5.10 & \\
\hline Elias 18 & 10.67 & 8.05 & 6.28 & 22.2 & -0.51 & $6.65 \pm 0.47$ & $14 \pm 1.4$ & $3.1 \pm 0.3$ & 5.35 & 1.3 & $\ldots$ & 3 \\
\hline ITG 25B & 12.41 & 10.25 & 8.87 & 12.5 & -0.50 & $4.63 \pm 0.57$ & $16 \pm 1.6$ & $2.0 \pm 0.2$ & 4.12 & 0.50 & $\ldots$ & 2 \\
\hline HH 47 IR & $\ldots$ & $\ldots$ & $\ldots$ & $\ldots$ & $\ldots$ & $23.91 \pm 0.24$ & $77.9 \pm 7.3$ & $15.6 \pm 1.6$ & 18.88 & $\ldots$ & 5.02 & 1 \\
\hline Ced 110 IRS6 & 20.99 & 14.74 & 10.92 & 46.0 & 0.66 & $12.97 \pm 0.31$ & $47 \pm 6$ & $26.3 \pm 4.0$ & 11.63 & 1.34 & $\ldots$ & 1,6 \\
\hline Cha II-28 & $\ldots$ & 13.90 & 10.66 & $\ldots$ & 0.89 & $5.71 \pm 0.25$ & $29.8 \pm 5.6$ & $\ldots$ & 5.03 & 0.67 & $\ldots$ & 1 \\
\hline CG 12 & 14.84 & $\ldots$ & 10.42 & $\ldots$ & 0.31 & $9.74 \pm 0.54$ & $20.7 \pm 2.0$ & $\ldots$ & 8.16 & 1.58 & $\ldots$ & 1 \\
\hline B228 & $\ldots$ & $\ldots$ & 14.32 & $\ldots$ & 1.82 & $50.5 \pm 0.5$ & $148 \pm 40$ & $\ldots$ & 9.71 & $\ldots$ & 40.80 & 1 \\
\hline Oph 125 & $\ldots$ & 14.69 & 10.56 & 51.6 & $\ldots$ & $4.91 \pm 0.51$ & $36.5 \pm 5$ & $3.9 \pm 0.6$ & 4.44 & 0.46 & $\ldots$ & 1 \\
\hline CRBR 2422.8-3423 & $\ldots$ & $\ldots$ & 13.68 & $\ldots$ & $\ldots$ & $13.50 \pm 0.20$ & $45 \pm 5$ & $31.1 \pm 4.0$ & 11.85 & 1.64 & $\ldots$ & 1 \\
\hline IRS 63 & 15.50 & 11.83 & 9.22 & 26.0 & 0.34 & $7.82 \pm 0.40$ & $20.4 \pm 3$ & $14.2 \pm 0.4$ & 6.27 & 1.55 & $\ldots$ & 1 \\
\hline CK 1 & 12.22 & 9.24 & 7.05 & 20.0 & $\ldots$ & $6.12 \pm 0.44$ & $16.9 \pm 1.6$ & $6.5 \pm 0.7$ & 4.72 & 1.39 & $\ldots$ & 1,4 \\
\hline R CrA IRS5 & .. & 13.65 & $\ldots$ & $\ldots$ & $\ldots$ & $18.26 \pm 0.22$ & $37.6 \pm 2.8$ & $6.3 \pm 0.4$ & 15.17 & 3.08 & $\ldots$ & 1,5 \\
\hline R CrA IRS7a & 12.55 & $\ldots$ & $\ldots$ & $\ldots$ & $\ldots$ & $23.50 \pm 0.23$ & $109 \pm 19$ & $10.0 \pm 1.0$ & 11.59 & $\ldots$ & 11.91 & 1,3 \\
\hline L723 & $\ldots$ & $\ldots$ & $\ldots$ & $\ldots$ & 0.50 & $49.0 \pm 4$ & $\ldots$ & $\ldots$ & 44.22 & 4.75 & $\ldots$ & 7 \\
\hline L1262 & $\ldots$ & 15.70 & 13.92 & $\ldots$ & 1.47 & $41.5 \pm 0.5$ & $130 \pm 23$ & $\ldots$ & 34.40 & 7.05 & $\ldots$ & 1 \\
\hline
\end{tabular}

Notes.

a All column densities are in units of $10^{-17} \mathrm{~cm}^{-2}$

${ }^{\mathrm{b}} \mathrm{H}_{2} \mathrm{O}$ and $\mathrm{CO}$ column densities are from (1) Pontoppidan et al. (2003, 2008); (2) unpublished IRTF data (J. E. Chiar et al. 2011, in preparation); (3) Chiar et al. (1998); (4) Chiar et al. (1994); (5) Tanaka et al. (1994). Additional notes: (6) Photometry for Ced 110 IRS6 is from Persi et al. (2001) (this source lacks reliable $J$ photometry from 2MASS). (7) The 2MASS point source closest to the position of L723 (J19175313+1912163) appears to be an unrelated field star.

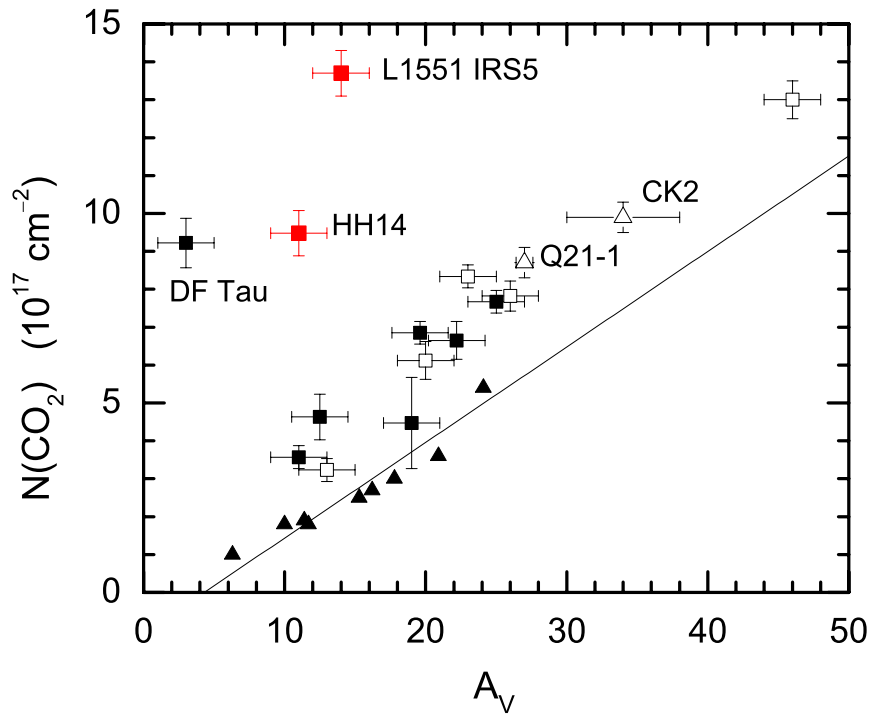

Figure 5. Plot of $\mathrm{CO}_{2}$ ice column density against visual extinction for YSOs from the present work (squares) and background field stars from Whittet et al. (2007, 2009; triangles). YSOs with P+CIM classification are filled in red; all other YSOs have $\mathrm{P}+\mathrm{NP}$ classification. Stars located in the direction of the Taurus dark cloud are distinguished by black filled symbols. The solid line is the linear least-squares fit to Taurus field stars only.

(A color version of this figure is available in the online journal.)
$N\left(\mathrm{CO}_{2}\right)$ data for YSOs, including our results (Table 3) and those from previous literature (Pontoppidan et al. 2008; Nummelin et al. 2001; Gerakines et al. 1999), together with field stars from Whittet et al. (2007, 2009). Figure 6 shows the overall correlation, Figure 7 is rescaled to show greater detail near the origin and with separate panels for all objects (above) and only Taurus objects (below). Taurus field stars show a particularly tight linear correlation $(R=0.99)$ passing through the origin to within statistical error:

$$
N\left(\mathrm{CO}_{2}\right)[\text { field }]=(0.18 \pm 0.04) N\left(\mathrm{H}_{2} \mathrm{O}\right)
$$

(Whittet et al. 2007; solid lines in Figures 6 and 7), indicating that these two species form in tandem in this quiescent cloud. The distribution of YSOs is also consistent with a linear correlation through the origin (a free fit yielding a negligible intercept); in this case, the correlation coefficient is $R=0.97$, and the slope is steeper in comparison to the correlation for Taurus field stars:

$$
N\left(\mathrm{CO}_{2}\right)[\mathrm{YSO}]=(0.29 \pm 0.01) N\left(\mathrm{H}_{2} \mathrm{O}\right)
$$

(dotted line in Figure 6 and the upper panel of Figure 7). Gerakines et al. (1999) found no significant dependence of the $N\left(\mathrm{CO}_{2}\right) / N\left(\mathrm{H}_{2} \mathrm{O}\right)$ ratio on environment in a smaller sample of predominantly high-mass YSOs and field stars, both falling 


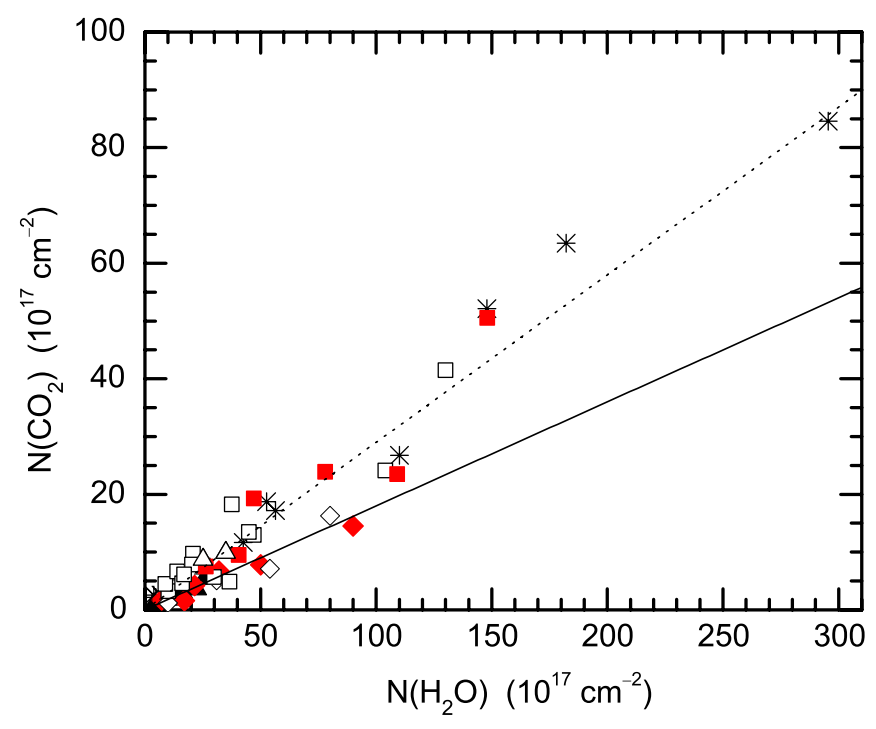

Figure 6. Plot of $N\left(\mathrm{CO}_{2}\right)$ vs. $N\left(\mathrm{H}_{2} \mathrm{O}\right)$ (see also Figure 7 for an enlargement of the region near the origin). Filled and open triangles denote field stars behind Taurus and other clouds, respectively (Whittet et al. 2007, 2009). All other symbols denote YSOs: P+NP YSOs from this paper (open squares); $\mathrm{P}+\mathrm{CIM}$ YSOs from this paper (red squares); P+NP YSOs from Gerakines et al. (1999, open diamonds); P+CIM YSOs from Gerakines et al. (1999, red diamonds); YSOs from Pontoppidan et al. (2008, asterisks); YSOs from Nummelin et al. (2001, crosses). The dotted and solid lines are linear least-squares fits to the correlations for all YSOs and Taurus field stars, respectively.

(A color version of this figure is available in the online journal.)

within essentially the same trend: $N\left(\mathrm{CO}_{2}\right) / N\left(\mathrm{H}_{2} \mathrm{O}\right)=0.17 \pm$ 0.03 . However, extended samples that include lower mass YSOs indicate that this ratio does display a degree of dependence on environment, our present work confirming the findings of Pontoppidan et al. (2008). The steeper slope of the YSO correlation line in comparison to the field-star correlation line in Figure 6 suggests enhanced production of $\mathrm{CO}_{2}$ in the environments of some YSOs. However, as a caveat, we note that $\mathrm{CO}_{2}$ abundances more in line with the YSO values are found for two non-Taurus field stars (CK2 in Serpens; in Q21-1 in IC5146; Knez et al. 2005; Whittet et al. 2009). The Taurus region is sufficiently well observed to allow a comparison of YSOs and field stars in the same cloud, and this is shown in Figure 7 (lower panel). This plot illustrates that, on average, Taurus YSOs have $\mathrm{CO}_{2}$ abundances a factor of $\sim 2$ higher relative to $\mathrm{H}_{2} \mathrm{O}$ compared with Taurus field stars.

Figure 8 shows the corresponding correlation between $N\left(\mathrm{H}_{2} \mathrm{O}\right)$ and $N(\mathrm{CO})$, again using all available data from the current work and previous literature cited above. The correlation lines for field stars and YSOs are

$$
\begin{aligned}
& N(\mathrm{CO})[\text { field }]=(0.47 \pm 0.1) N\left(\mathrm{H}_{2} \mathrm{O}\right)-(3.16 \pm 2.0) \\
& N(\mathrm{CO})[\mathrm{YSO}]=(0.12 \pm 0.1) N\left(\mathrm{H}_{2} \mathrm{O}\right)+(2.31 \pm 2.0) .
\end{aligned}
$$

In this case, the YSOs show a much greater degree of scatter compared with field stars (the correlation coefficients are $R=$ 0.45 and 0.89 , respectively). As $\mathrm{CO}$ is more volatile than $\mathrm{H}_{2} \mathrm{O}$ or $\mathrm{CO}_{2}$, it is expected to sublimate far more readily upon heating. The data in Figure 8 are consistent with varying degrees of $\mathrm{CO}$ sublimation, determined by the local environment in the vicinity of each YSO. This conclusion is supported by the fact that YSOs in the P+CIM group tend to have systematically lower CO column densities compared with the average for all YSOs.

Column density estimates for the components of our profile fits may be used to investigate their intercorrelation. Our fitting

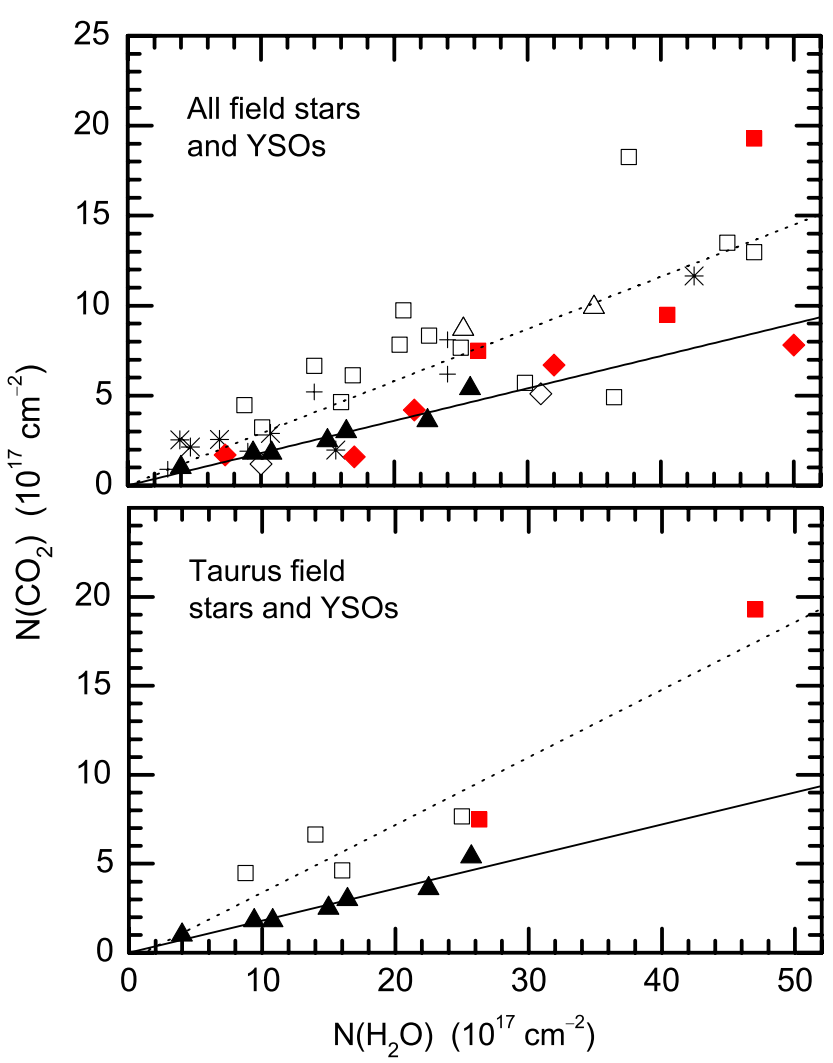

Figure 7. Plots of $N\left(\mathrm{CO}_{2}\right)$ vs. $N\left(\mathrm{H}_{2} \mathrm{O}\right)$, similar to Figure 6 , but rescaled to show greater detail near the origin and with separate panels for all objects (above) and only Taurus objects (below). Symbols have the same meaning as in Figure 6. The dotted lines are fits to all YSOs and Taurus YSOs in the upper and lower panels, respectively; the solid line in each panel is the fit to Taurus field stars. (A color version of this figure is available in the online journal.)

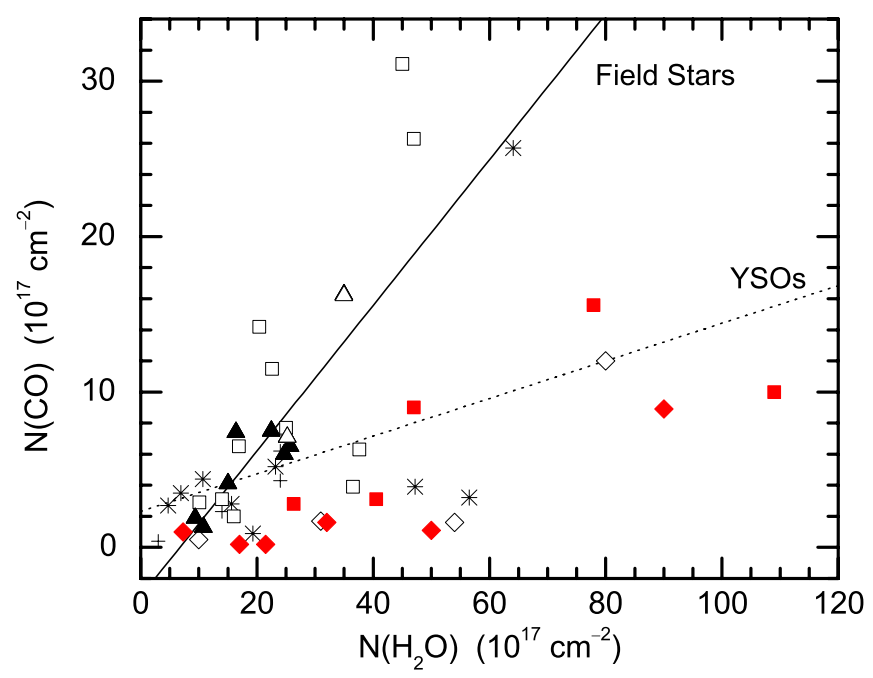

Figure 8. Plot of $N(\mathrm{CO})$ vs. $N\left(\mathrm{H}_{2} \mathrm{O}\right)$. Symbols have the same meaning as in Figure 6. The dotted and solid lines are the least-squares fits to the correlations for YSOs and field stars, respectively.

(A color version of this figure is available in the online journal.)

procedure (Section 3.2) assumes that two of three possible mixtures (cold polar, cold nonpolar, and warmed ices) are contributing to the observed $15 \mu \mathrm{m}$ feature, and that all lines of sight include a polar component. The four panels of Figure 9 plot $N\left(\mathrm{CO}_{2}\right)$ values for each relevant component and for the total (Table 3), in each case against $N\left(\mathrm{H}_{2} \mathrm{O}\right)$. The parameters of the 

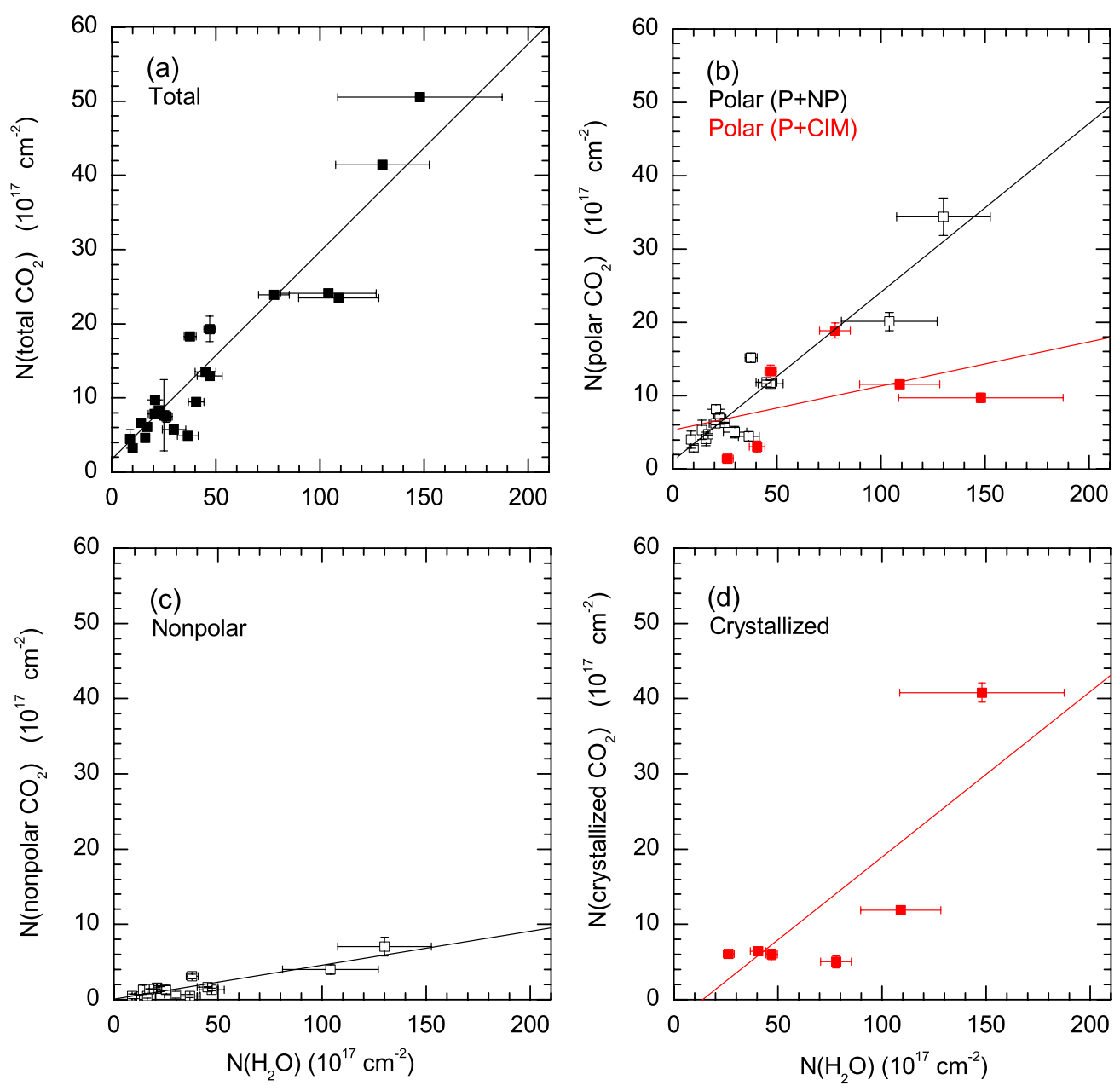

Figure 9. Correlations of $N\left(\mathrm{CO}_{2}\right)$ ice components with $N\left(\mathrm{H}_{2} \mathrm{O}\right)$, based on our laboratory fits to the $15 \mu \mathrm{m} \mathrm{CO}_{2}$ feature for YSOs in our sample (Tables 2 and 3). The panels represent (a) total, (b) polar, (c) nonpolar, and (d) crystallized $\mathrm{CO}_{2}$. Red squares and black open squares represent $\mathrm{P}+\mathrm{CIM}$ sources and $\mathrm{P}+\mathrm{NP}$ sources, respectively; black squares represent the total (polar, nonpolar, crystallized) $\mathrm{CO}_{2}$ for all sources. Lines represent the linear least-squares fit for each correlation; in panel (b), the black and red lines are fits to $\mathrm{P}+\mathrm{NP}$ and $\mathrm{P}+\mathrm{CIM}$ data, respectively.

(A color version of this figure is available in the online journal.)

Table 4

Correlation Lines for Figure 9

\begin{tabular}{lcccl}
\hline \hline $\begin{array}{c}\text { Correlation Line } \\
N\left(\mathrm{H}_{2} \mathrm{O}\right) \text { versus }\end{array}$ & Slope & Intercept & $\begin{array}{c}\text { Correlation } \\
\text { Coefficient }\end{array}$ & Panel in Figure 9 \\
\hline Total $\mathrm{CO}_{2}$ & $0.28 \pm 0.03$ & $1.72 \pm 1.6$ & 0.95 & $\mathrm{a}$ \\
Polar $\mathrm{CO}_{2}, \mathrm{P}+\mathrm{NP}$ only & $0.23 \pm 0.02$ & $1.16 \pm 1.0$ & 0.94 & b, black line \\
Polar $\mathrm{CO}_{2}, \mathrm{P}+\mathrm{CIM}$ only & $0.06 \pm 0.06$ & $5.35 \pm 5.5$ & 0.41 & b, red line \\
Nonpolar $\mathrm{CO}_{2}$ & $0.04 \pm 0.01$ & $0.09 \pm 0.3$ & 0.90 & $\mathrm{c}$ \\
Crystallized $\mathrm{CO}_{2}$ & $0.22 \pm 0.07$ & $-3.06 \pm 5.5$ & 0.84 & $\mathrm{~d}$ \\
\hline
\end{tabular}

Note. ${ }^{\text {a }}$ Format: $N\left(\mathrm{CO}_{2}\right)=c_{1} N\left(\mathrm{H}_{2} \mathrm{O}\right)+c_{2}$, where $c_{1}$ and $c_{2}$ are the slope and intercept.

fits are listed in Table 4. Figure 9 (panel (a)) confirms the strong correlation between $N\left(\mathrm{H}_{2} \mathrm{O}\right)$ and total $N\left(\mathrm{CO}_{2}\right)$ for our sample, as expected on the basis of Figure 6. A high degree of correlation is also seen in both the polar and nonpolar components of the $\mathrm{P}+\mathrm{NP}$ sources individually (panels (b) and (c) of Figure 9). This is in contrast to the situation for the $\mathrm{P}+\mathrm{CIM}$ group, which display greater scatter and a trend in $\mathrm{CO}_{2}$ abundance toward lower concentrations in the polar ice component and relatively high concentrations in the crystallized component (panels (b) and $(\mathrm{d}))$.

The origin of the crystallized ice component is an important question (see Pontoppidan et al. 2008 for detailed discussion).
The apparent reduction in polar ices toward some YSOs with crystalline components (Figure 9) is consistent with a scenario in which it is predominantly the polar ices that are being crystallized; the total abundance of $\mathrm{CO}_{2}$ in the mantles is conserved during the crystallization process, with scatter arising from different levels of thermal processing in each line of sight. The polar ices are, indeed, the primary reservoir of $\mathrm{CO}_{2}$ in the ices and high abundances of crystalline $\mathrm{CO}_{2}$ can only arise if that reservoir is tapped. However, the laboratory CIM analogs used in our fits are presumed to be only partially crystallized, and the total abundance of crystallized $\mathrm{CO}_{2}$ needed to explain the subfeatures in the profiles may thus be relatively small. 
Pontoppidan et al. (2008) propose that crystallization of $\mathrm{CO}_{2}$ accompanies distillation of the nonpolar ices as $\mathrm{CO}$ sublimates, which may be more probable in the envelopes of low-luminosity YSOs as it can occur at substantially lower temperatures $(20-40 \mathrm{~K})$ than those required for thermal processing of the polar ices $(>50 \mathrm{~K})$. Our results do not provide a definitive answer to this question but they do suggest that the polar ices may contribute to crystalline $\mathrm{CO}_{2}$.

\subsection{The Ternary Plot}

As previously noted, observations show $\mathrm{H}_{2} \mathrm{O}, \mathrm{CO}$, and $\mathrm{CO}_{2}$ to be the most abundant constituents of ices in molecular clouds. A ternary plot is a useful analytical tool for studying a system with three primary constituents, allowing a two-dimensional representation of the relative contributions of each to the total. ${ }^{10}$ Figure 10 presents ternary plots for the $\mathrm{H}_{2} \mathrm{O}-\mathrm{CO}-\mathrm{CO}_{2}$ system in interstellar ices (plots displaying all available data and data for the Taurus region only are shown). A pure ice composed entirely of one of these molecules would plot at the relevant vertex of the triangle (left, right, or top vertex for $\mathrm{CO}, \mathrm{H}_{2} \mathrm{O}$, or $\mathrm{CO}_{2}$, respectively). The scales on the sides of the triangle denote relative abundances, and the values for a given point should be read along lines parallel to the tick marks for each axis. As $\mathrm{H}_{2} \mathrm{O}$ is by far the most abundant of the three, it is scaled by a factor of 0.2 so that an "average" ice composition falls near the center of the plot (rather than near the $\mathrm{H}_{2} \mathrm{O}$ vertex). Each plotted point represents a line of sight in which reliable data exist for all three constituents, its position determined by the fractional abundance of each relative to the sum. YSOs from the present paper and from Pontoppidan et al. (2008) are included, together with field stars from our previous work (Whittet et al. 2007, 2009). Field stars and YSOs (P+NP or P+CIM classification) are distinguished by plotting symbol. Examples of possible evolutionary trends are illustrated by the insets: variation in $\mathrm{CO}$ concentration independent of $\mathrm{H}_{2} \mathrm{O}: \mathrm{CO}_{2}$ ratio, as might arise from thermal processing of nonpolar ices (I); and variation in $\mathrm{H}_{2} \mathrm{O}: \mathrm{CO}_{2}$ ratio independent of $\mathrm{CO}$ concentration, as might arise from differences in the surface chemistry that forms the polar ices (II).

Background field stars (triangles) in Figure 10 follow a distribution broadly consistent with variations in $\mathrm{CO}$ abundance independent of the relative proportions of the other two constituents. As most of the $\mathrm{CO}$ in a typical line of sight resides in the volatile nonpolar component of the mantles, this distribution can be interpreted as a trend of condensation versus sublimation of nonpolar ices (diagonal trend toward and away from the $\mathrm{CO}$ vertex of the plot, respectively, as indicated by the dotted lines and inset I in Figure 10). The loci of individual field stars along the trend correlate loosely with $A_{V}$, reflecting a greater preponderance of $\mathrm{CO}$ in the mantles for lines of sight containing denser (more optically thick) regions within the molecular clouds. Scatter orthogonal to the trend indicates variations in $\mathrm{CO}_{2}: \mathrm{H}_{2} \mathrm{O}$ ratio. Comparison of the field-star and YSO distributions reveals both similarities and differences. The distribution of $\mathrm{P}+\mathrm{NP}$ YSOs (black squares) generally resembles

\footnotetext{
10 The ternary plot provides a more objective analysis than (say) examining the $x-y$ plot of $\mathrm{CO} / \mathrm{H}_{2} \mathrm{O}$ versus $\mathrm{CO}_{2} / \mathrm{H}_{2} \mathrm{O}$, which effectively assumes that $N\left(\mathrm{H}_{2} \mathrm{O}\right)$ scales with total ice column and that the ratios of $\mathrm{CO}$ and $\mathrm{CO}_{2}$ to $\mathrm{H}_{2} \mathrm{O}$ represent the abundances of these two molecules in the ices. This can lead to confusion between cloud-to-cloud variations and global trends in ice properties: Serpens provides a well-documented example of a cloud where systematic differences occur in the abundance of $\mathrm{H}_{2} \mathrm{O}$ relative to other ices in comparison to other clouds (Whittet et al. 2009 and references therein).
}
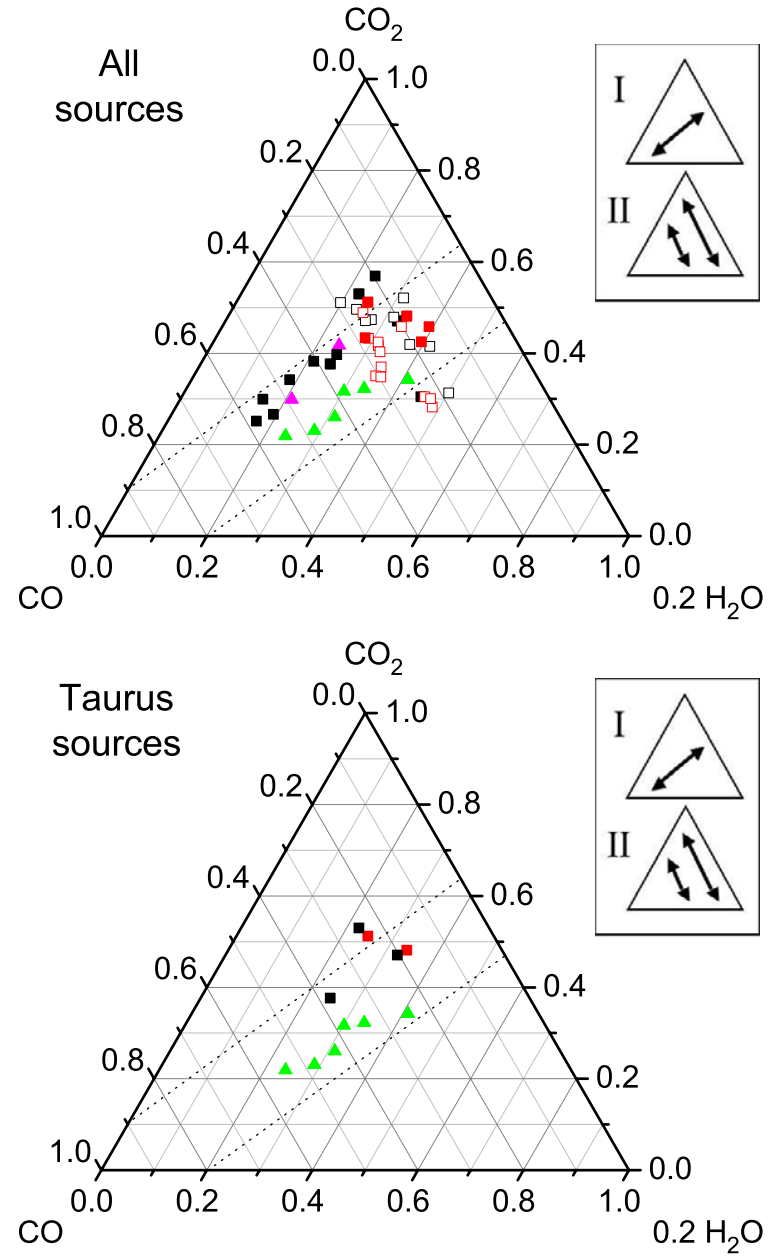

Figure 10. Ternary diagrams for $\mathrm{H}_{2} \mathrm{O}, \mathrm{CO}$, and $\mathrm{CO}_{2}$ in interstellar ices. All available data are plotted in the upper panel; the lower panel is limited to lines of sight in the Taurus region. The $\mathrm{H}_{2} \mathrm{O}$ axis is scaled by a factor of 0.2 so that ices with average composition plot near the center of each plot. Triangles denote field stars behind Taurus (green) and other clouds (magenta) (Whittet et al. 2007, 2009). Squares denote YSOs from the current work (filled) and Pontoppidan et al. (2008, open), colored red in cases where the $\mathrm{CO}_{2}$ profile indicates a crystallized ice component. Examples of possible evolutionary trends are illustrated by the insets. See Section 5.3 for further explanation and discussion.

(A color version of this figure is available in the online journal.)

that of field stars, i.e., consistent with a trend dominated by $\mathrm{CO}$ condensation/sublimation, but with a tendency for greater scatter as the $\mathrm{CO}$ abundance becomes smaller. P+CIM YSOs (red squares) are segregated away from the $\mathrm{CO}$ vortex, as expected given that they show independent evidence for thermal processing: the heating responsible for crystallization seems likely to result in simultaneous (partial) sublimation of the volatile nonpolar CO-rich ice component.

The vertical spread in the points suggests variations in $\mathrm{CO}_{2}$ concentration, which may arise in (at least) two different ways: it may reflect (1) differences in $\mathrm{CO}_{2}$ production rates at low temperature within the parent molecular cloud, or (2) formation of $\mathrm{CO}_{2}$ by energetic processes in the vicinity of YSOs. Models suggest that the composition of the ices in a molecular cloud depends on both physical conditions and evolutionary state (e.g., Ruffle \& Herbst 2001; Garrod et al. 2008). Formation of $\mathrm{CO}_{2}$ is thought to proceed principally via grain surface reactions such as $\mathrm{CO}+\mathrm{O} \rightarrow \mathrm{CO}_{2}$ and $\mathrm{CO}+\mathrm{OH} \rightarrow \mathrm{CO}_{2}+\mathrm{H}$. A cloud with relatively high gaseous $\mathrm{CO}$ abundance (determined by 
gas-phase chemistry) may thus tend to form ices containing more $\mathrm{CO}_{2}$ (as well as $\mathrm{CO}$ ) relative to $\mathrm{H}_{2} \mathrm{O}$. Observed differences in $\mathrm{CO}_{2}: \mathrm{H}_{2} \mathrm{O}$ concentration in the Serpens and IC 5146 dark clouds compared with Taurus might be explained in this way (Whittet et al. 2009). The onset of star formation enables another route to $\mathrm{CO}_{2}$ : it is well known that exposure of interstellar ice analogs to energetic radiation promotes $\mathrm{CO}_{2}$ production in the laboratory (e.g., Ioppolo et al. 2009 and references therein).

In order to distinguish between systemic cloud-to-cloud differences in $\mathrm{CO}_{2}$ production efficiency and production driven by the individual YSOs they contain, it is informative to compare lines of sight within individual clouds. Unfortunately, at present, only Taurus has sufficient coverage for a meaningful comparison: all available data for this region are plotted in the right-hand frame of Figure 10. It is notable that the five YSOs in this sample all lie above the trend for field stars. This vertical separation suggests that each YSO has a significant local component, as well as a molecular-cloud component, to its total dust column, and that $\mathrm{CO}_{2}$ is being produced in the local component by processes driven by the YSO itself. This effect is seen in both $\mathrm{P}+\mathrm{NP}$ and $\mathrm{P}+\mathrm{CIM}$ YSOs, suggesting that energetic $\mathrm{CO}_{2}$ production is not strongly correlated with the heating responsible for crystallization.

To summarize, it appears that the distribution of points in Figure 10 can be explained by the following.

1. Variation in $\mathrm{CO}_{2}$ production efficiency that results from differences in the chemistry and/or evolutionary state of the parent molecular cloud.

2. A trend of condensation versus sublimation of CO-rich ices that culminates in crystallization of the ices toward YSOs with the highest degrees of thermal processing.

3. Enhanced $\mathrm{CO}_{2}$ production by energetic processes in the vicinity of YSOs.

\subsection{Correlation with YSO Spectral Index}

To provide an evolutionary classification for each YSO, we estimate the spectral index $\alpha$ represented by the mean slope of the spectral energy distribution (SED),

$$
\alpha \equiv \frac{d \log \lambda F_{\lambda}}{d \log \lambda} \approx \frac{\log \lambda_{2} F_{\lambda_{2}}-\log \lambda_{1} F_{\lambda_{1}}}{\log \lambda_{2}-\log \lambda_{1}},
$$

where $\lambda_{1}=2.15 \mu \mathrm{m}$ and $\lambda_{2}=25 \mu \mathrm{m}$ (e.g., Kenyon $\&$ Hartmann 1995). Fluxes are taken from the point-source catalogs of the 2MASS and the Infrared Astronomical Satellite, respectively. Values of $\alpha$ for our sources are reported in Table 3. In general, a more positive SED slope (larger $\alpha$ ) corresponds to a younger, more deeply embedded YSO with a cool dust envelope and a high column density of molecular-cloud material. As the YSO evolves the envelope dissipates and $\alpha$ is reduced, approaching a value of -3 in the Rayleigh-Jeans limit.

Figure 11 displays plots of $\alpha$ versus $N\left(\mathrm{H}_{2} \mathrm{O}\right), N\left(\mathrm{CO}_{2}\right)$, and the ratio $N\left(\mathrm{CO}_{2}\right) / N\left(\mathrm{H}_{2} \mathrm{O}\right)$. As expected, the individual column densities $N\left(\mathrm{H}_{2} \mathrm{O}\right)$ and (to a lesser degree) $N\left(\mathrm{CO}_{2}\right)$ correlate positively with $\alpha$, indicating larger ice columns toward more deeply embedded YSOs. These trends are consistent with those found by Zasowski et al. (2009, their Figure 13) for a smaller sample of low-mass YSOs in the Taurus region. The $\alpha$ versus $N\left(\mathrm{CO}_{2}\right) / N\left(\mathrm{H}_{2} \mathrm{O}\right)$ data (lower panel) are uncorrelated or weakly anticorrelated. Several YSOs display $\mathrm{CO}_{2}$ concentrations in excess of the range occupied by field stars sampling dark clouds (indicated by horizontal dotted lines in the lower panel of Figure 11), a difference that tends to become more pronounced
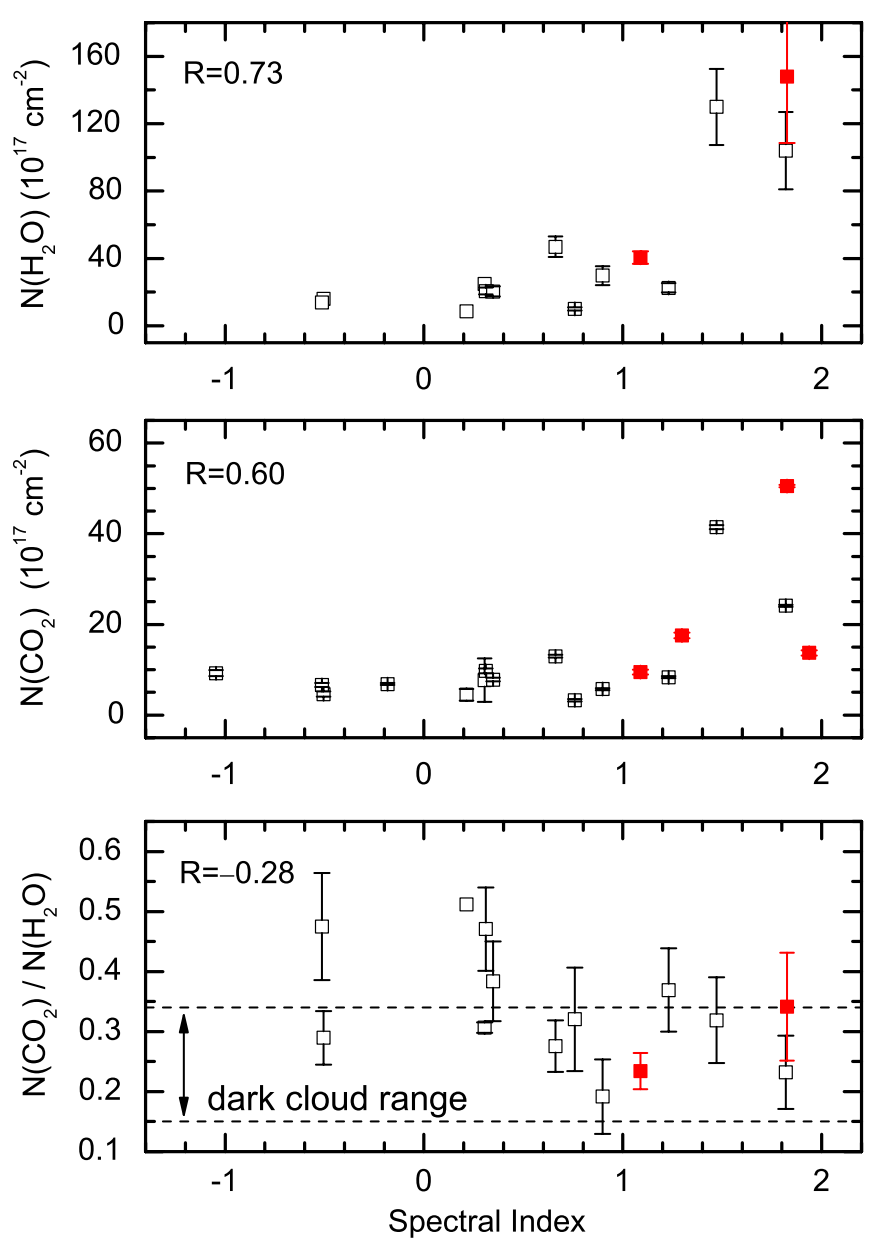

Figure 11. Plots of spectral index vs. $\mathrm{CO}_{2}$ and $\mathrm{H}_{2} \mathrm{O}$ column densities (top and middle frames) and the ratio between them (bottom frame) for YSOs in our sample. Open squares and red squares are P+NP YSOs and P+CIM YSOs, respectively. Horizontal dashed lines in the bottom panel delineate the $N\left(\mathrm{CO}_{2}\right)$ / $N\left(\mathrm{H}_{2} \mathrm{O}\right)$ range observed in field stars toward dark clouds (Taurus, Serpens, and IC5146; Whittet et al. 2007, 2009).

(A color version of this figure is available in the online journal.)

for lower values of $\alpha$, consistent with a trend toward higher $\mathrm{CO}_{2}$ abundances in ices toward more evolved YSOs. These results provide further tentative support for enhanced $\mathrm{CO}_{2}$ production in the environments of some YSOs.

\subsection{The Partition of Oxygen between Polar and Nonpolar Ices}

Previous observations of background field stars suggest that the abundance of elemental oxygen in the ices in the cold, dense interstellar medium shows consistent behavior from cloud to cloud, and that the distribution of the oxygen between polar and nonpolar components is almost invariant in such regions (Whittet et al. 2009). The extent to which these findings apply to lines of sight toward YSOs is investigated here. Again, we begin by assuming that the three molecules $\mathrm{H}_{2} \mathrm{O}, \mathrm{CO}$, and $\mathrm{CO}_{2}$ represent a nearly complete inventory of oxygen reservoirs in the ices (see Whittet et al. 2007, 2009 for discussion and caveats). The column densities of elemental oxygen sequestered into polar and nonpolar mantles is then given by

$$
\begin{gathered}
N(\mathrm{O})_{\text {polar }}=N\left(\mathrm{H}_{2} \mathrm{O}\right)+f_{1} N(\mathrm{CO})+2 f_{2} N\left(\mathrm{CO}_{2}\right) \\
N(\mathrm{O})_{\text {nonpolar }}=\left(1-f_{1}\right) N(\mathrm{CO})+2\left(1-f_{2}\right) N\left(\mathrm{CO}_{2}\right),
\end{gathered}
$$

where $f_{1}$ and $f_{2}$ are the fractions of all solid-phase $\mathrm{CO}$ and $\mathrm{CO}_{2}$ molecules, respectively, in the polar ices. Values of $f_{1}$ are 


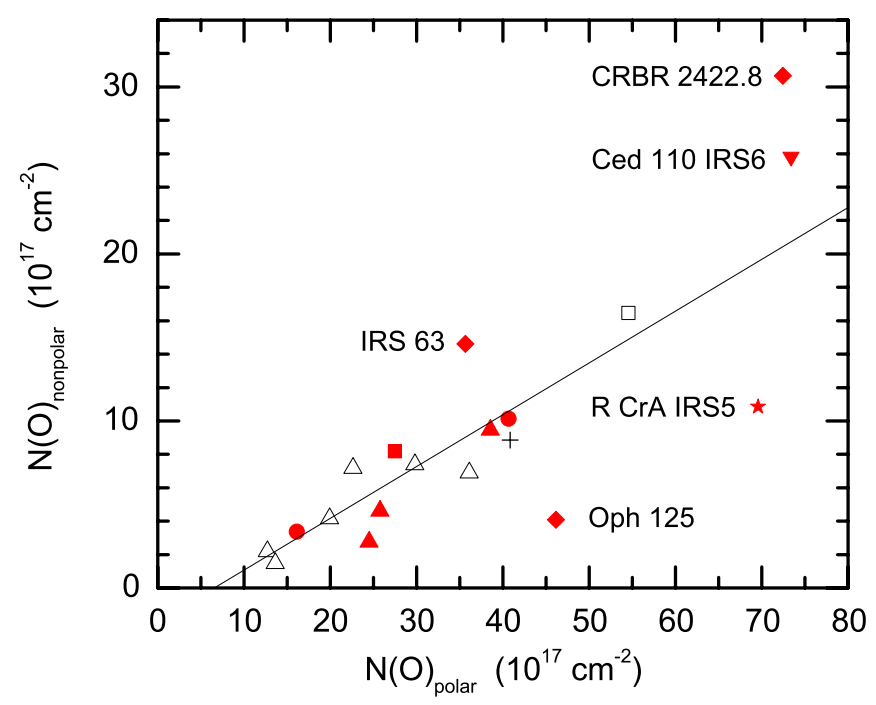

Figure 12. Plot of the estimated column density of elemental oxygen in the polar and nonpolar ices. Black symbols represent field stars from Whittet et al. (2009) in Taurus (triangles), Serpens (square), and IC5146 (plus sign). Red symbols represent YSOs in Taurus (upright triangles), Serpens (square), $\rho$ Oph (diamonds), Perseus (circles), Chameleon (inverted triangle), and CrA (star). The line is the linear least-squares fit to field stars only.

(A color version of this figure is available in the online journal.)

taken from previous work (Chiar et al. 1994, 1995, 2011; Kerr et al. 1993) where $f_{1}=0.17$ for Serpens, 0.12 for Taurus and IC 5146, and 0.19 for $\rho \mathrm{Oph}$; for clouds with no direct measurement, we adopt the Taurus value. The parameter $f_{2}$ is evaluated using the $\chi_{v}^{2}$ fitting results of the present paper for YSOs and of Whittet et al. (2007, 2009) for field stars. The average values of $f_{2}$ are 0.73 for YSOs and 0.85 for field stars.

Figure 12 plots $N(\mathrm{O})_{\text {nonpolar versus }} N(\mathrm{O})_{\text {polar }}$ for YSOs and field stars. The correlation line shown is for field stars only: although YSOs show greater scatter, the majority are nevertheless consistent with the field-star trend. Preferential loss of nonpolar mantles (distribution below the correlation line) is expected in situations where ices are warmed significantly: pure $\mathrm{CO}$, for example, will sublimate at $T>17 \mathrm{~K}$. The opposite effect where nonpolar ices tend to dominate (distribution above the correlation line) may arise in the coldest, densest clouds or in situations where gas-phase chemistry favors efficient $\mathrm{CO}$ production. These processes might readily explain the loci of certain YSOs. It is notable that three of the most extreme cases lie in the same cloud ( $\rho$ Oph: CRBR 2422.8-3423, IRS 63 and Oph 125). This cloud is known to contain relatively steep temperature gradients, from the coldest cores to relatively warm environments subject to a local radiation field enhanced by the presence of nearby luminous stars (Liseau et al. 1999; Di Francesco et al. 2004).

\section{CONCLUSIONS}

We have reduced and analyzed $10-19.5 \mu \mathrm{m}$ spectra from the Spitzer archive for 28 YSOs exhibiting $\mathrm{CO}_{2}$ bendingmode ice absorption features, and characterized them by fitting model spectra using data for laboratory analogs with different compositions and temperatures. Modeling leads to classification of the spectra into two groups: $\mathrm{P}+\mathrm{NP}$ and $\mathrm{P}+\mathrm{CIM}$. YSOs in the $\mathrm{P}+\mathrm{NP}$ group exhibit simple $\mathrm{CO}_{2}$ absorption profiles resembling those of field stars such as Elias 16, consistent with ices at low temperatures (typically 10-20 K) and dominated by the polar ice component. YSOs in the P+CIM group exhibit structure in the profiles consistent with the presence of ices formed or modified at temperatures substantially higher than those prevailing in cold molecular clouds.

Analyses of data on the abundances of the three principal molecules $\left(\mathrm{H}_{2} \mathrm{O}, \mathrm{CO}\right.$, and $\left.\mathrm{CO}_{2}\right)$ present in the ices suggests systematic variations in composition determined by initial conditions and/or subsequent evolution. Variations in $\mathrm{CO}_{2}$ production efficiency resulting from differences in the chemistry and/or evolutionary state of the parent molecular cloud plays a role in determining the abundance of $\mathrm{CO}_{2}$ in the ices prior to any further evolution in the vicinity of YSOs. The abundance of $\mathrm{CO}_{2}$ is typically enhanced in lines of sight to YSOs compared with those toward field stars sampling quiescent regions of the prototypical (Taurus) molecular cloud. Thermal or energetic processing driven by the YSOs themselves may thus be responsible for enhanced production of $\mathrm{CO}_{2}$. Warming causes sublimation of $\mathrm{CO}$ from the ices in the lines of sight to many YSOs, a trend that culminates in crystallization of the ices toward those with the highest degrees of thermal processing. The origin of the crystallized ice component remains an important unanswered question: Pontoppidan et al. (2008) propose a distillation process involving the nonpolar ices; our fits suggest a redistribution of $\mathrm{CO}_{2}$ from polar to crystalline ices toward some YSOs, consistent with thermal processing of the more abundant polar component.

The ternary plot appears to be a valuable analytical tool for studying interstellar ices. To fully exploit its potential, it will be important in the future to obtain reliable data for $\mathrm{H}_{2} \mathrm{O}, \mathrm{CO}$, and $\mathrm{CO}_{2}$ in a greater number of lines of sight, with adequate sampling of both field stars and YSOs in representative molecular clouds and star formation regions covering a range of evolutionary states. The $\mathrm{H}_{2} \mathrm{O}, \mathrm{CO}_{2}$, and $\mathrm{CH}_{3} \mathrm{OH}$ system may also be a valuable one to study with this analytical approach, as $\mathrm{CO}_{2}$ and $\mathrm{CH}_{3} \mathrm{OH}$ represent alternative routes for $\mathrm{CO}$ chemistry in the environments where $\mathrm{H}_{2} \mathrm{O}$-rich ices form. Again this will require acquisition of new data, specifically to extend the database of $\mathrm{CH}_{3} \mathrm{OH}$ detections toward sources with existing data for $\mathrm{CO}_{2}$.

This work is based on observations made with the Spitzer Space Telescope, which is operated by the Jet Propulsion Laboratory, California Institute of Technology, under a contract with NASA. Financial support for this research was provided by the Spitzer General Observer and Archival Research Programs (JPL/Caltech Support Agreements nos. 1264149 and 1290823), the NASA Exobiology and Evolutionary Biology program (grant NNX07AK38G), the NASA Astrobiology Institute (grant NNA09DA80A), and the NASA New York Space Grant Consortium. We are grateful to an anonymous referee for helpful comments.

Facilities: Spitzer (IRS), IRTF (SpeX), FLWO:2MASS, CTIO:2MASS

\section{REFERENCES}

Bergin, E. A., Melnick, G. J., Gerakines, P. A., Neufeld, D. A., \& Whittet, D. C. B. 2005, ApJ, 627, L33

Boogert, A. C. A. 1999, PhD thesis, Univ. Groningen

Boogert, A. C. A., et al. 2004, ApJS, 154, 359

Boogert, A. C. A., et al. 2008, ApJ, 678, 985

Chiar, J. E., Adamson, A. J., Kerr, T. H., \& Whittet, D. C. B. 1994, ApJ, 426, 240

Chiar, J. E., Adamson, A. J., Kerr, T. H., \& Whittet, D. C. B. 1995, ApJ, 455, 234

Chiar, J. E., et al. 1998, ApJ, 498, 716 
Chiar, J. E., et al. 2011, ApJ, in press

Cuppen, H. M., van Dishoeck, E. F., Herbst, E., \& Tielens, A. G. G. M. 2009, A\&A, 508, 275

Dartois, E., Demyk, K., d'Hendecourt, L., \& Ehrenfreund, P. 1999, A\&A, 351, 1066

d'Hendecourt, L. B., Allamandola, L. J., Grim, R. J. A., \& Greenberg, J. M. 1986, A\&A, 158, 119

Di Francesco, J., André, P., \& Myers, P. C. 2004, ApJ, 617, 425

Ehrenfreund, P., Boogert, A. C. A., Gerakines, P. A., Tielens, A. G. G. M., \& van Dishoeck, E. F. 1997, A\&A, 328, 649

Ehrenfreund, P., Dartois, E., Demyk, K., \& d'Hendecourt, L. 1998, A\&A, 339, L17

Ehrenfreund, P., et al. 1999, A\&A, 350, 240

Garrod, R. T., Weaver, S. L. W., \& Herbst, E. 2008, ApJ, 682, 283

Gerakines, P. A., Schutte, W. A., Greenberg, J. M., \& van Dishoeck, E. F. 1995 A\&A, 296, 810

Gerakines, P. A., et al. 1999, ApJ, 522, 357

Gibb, E. L., Whittet, D. C. B., Boogert, A. C. A., \& Tielens, A. G. G. M. 2004, ApJS, 151, 35

Gibb, E. L., et al. 2000, ApJ, 536, 347

Herbst, E., \& van Dishoeck, E. F. 2009, ARA\&A, 47, 427

Houck, J. R., et al. 2004, ApJS, 154, 18

Ioppolo, S., Palumbo, M. E., Baratta, G. A., \& Mennella, V. 2009, A\&A, 493, 1017

Kenyon, S. J., \& Hartmann, L. 1995, ApJS, 101, 117

Kerr, T. H., Adamson, A. J., \& Whittet, D. C. B. 1993, MNRAS, 262, 1047

Knez, C., et al. 2005, ApJ, 635, L145

Lamzin, S. A., Melnikov, S. Y., Grankin, K. N., \& Ezhkova, O. V. 2001, A\&A, 372,922

Langer, W. D., van Dishoeck, E. F., Bergin, E. A., Blake, G. A., Tielens, A. G. G. M., Velusamy, T., \& Whittet, D. C. B. 2000, in Protostars and Planets IV, ed. V. Mannings, A. P. Boss, \& S. S. Russell (Tucson, AZ: Univ. Arizona Press), 29

Li, J. Z., Ip, W. H., Chen, W. P., Hu, J. Y., \& Wei, J. Y. 2001, ApJ, 549, L89
Liseau, R., et al. 1999, A\&A, 344, 342

Martin, P. G., \& Whittet, D. C. B. 1990, ApJ, 357, 113

Moore, M. H., Ferrante, R. F., Moore, W. J., \& Hudson, R. 2010, ApJS, 191, 96

Nummelin, A., Whittet, D. C. B., Gibb, E. L., Gerakines, P. A., \& Chiar, J. E. 2001, ApJ, 558, 185

Oba, Y., Watanabe, N., Kouchi, A., Hama, T., \& Pirronello, V. 2010, ApJ, 712, L174

Persi, P., Marenzi, A. R., Gómez, M., \& Olofsson, G. 2001, A\&A, 376, 907

Pontoppidan, K. M., Dullemond, C. P., van Dishoeck, E. F., Blake, G. A., Boogert, A. C. A., Evans, N. J., Kessler-Silacci, J. E., \& Lahuis, F. 2005, ApJ, 622,463

Pontoppidan, K. M., et al. 2003, A\&A, 408, 981

Pontoppidan, K. M., et al. 2008, ApJ, 678, 1005

Ruffle, D. P., \& Herbst, E. 2001, MNRAS, 324, 1054

Shenoy, S. S., Whittet, D. C. B., Ives, J. A., \& Watson, D. M. 2008, ApJS, 176, 457

Skrutskie, M. F., et al. 2006, AJ, 131, 1163

Tanaka, M., Nagata, T., Sato, S., \& Yamamoto, T. 1994, ApJ, 430, 779

Tielens, A. G. G. M., Tokunaga, A. T., Geballe, T. R., \& Baas, F. 1991, ApJ, 381,181

van Broekhuizen, F. A., Groot, I. M. N., Fraser, H. J., van Dishoeck, E. F., \& Schlemmer, S. 2006, A\&A, 451, 723

Watson, D. M., et al. 2004, ApJS, 154, 391

White, D. W., Gerakines, P. A., Cook, A. M., \& Whittet, D. C. B. 2009, ApJS, 180,182

Whittet, D. C. B., Cook, A. M., Chiar, J. E., Pendleton, Y. J., Shenoy, S. S., \& Gerakines, P. A. 2009, ApJ, 695, 94

Whittet, D. C. B., Gerakines, P. A., Hough, J. H., \& Shenoy, S. S. 2001, ApJ, 547,872

Whittet, D. C. B., Shenoy, S. S., Bergin, E. A., Chiar, J. E., Gerakines, P. A., Gibb, E. L., Melnick, G. J., \& Neufeld, D. A. 2007, ApJ, 655, 332

Whittet, D. C. B., et al. 1998, ApJ, 498, L159

Zasowski, G., Kemper, F., Watson, D. M., Furlan, E., Bohac, C. J., Hull, C., \& Green, J. D. 2009, ApJ, 694, 459 